Review Article

\title{
Recent Developments in the X-Ray Reflectivity Analysis for Rough Surfaces and Interfaces of Multilayered Thin Film Materials
}

\author{
Yoshikazu Fujii \\ Center for Supports to Research and Education Activities, Kobe University, Kobe 657-8501, Japan \\ Correspondence should be addressed to Yoshikazu Fujii; fujiiyos@kobe-u.ac.jp
}

Received 31 December 2012; Revised 6 June 2013; Accepted 24 June 2013

Academic Editor: Te-Hua Fang

Copyright (C) 2013 Yoshikazu Fujii. This is an open access article distributed under the Creative Commons Attribution License, which permits unrestricted use, distribution, and reproduction in any medium, provided the original work is properly cited.

\begin{abstract}
$\mathrm{X}$-ray reflectometry is a powerful tool for investigations on rough surface and interface structures of multilayered thin film materials. The X-ray reflectivity has been calculated based on the Parratt formalism, accounting for the effect of roughness by the theory of Nevot-Croce conventionally. However, in previous studies, the calculations of the X-ray reflectivity often show a strange effect where interference effects would increase at a rough surface. And estimated surface and interface roughnesses from the X-ray reflectivity measurements did not correspond to the TEM image observation results. The strange result had its origin in a used equation due to a serious mistake in which the Fresnel transmission coefficient in the reflectivity equation is increased at a rough interface because of a lack of consideration of diffuse scattering. In this review, a new accurate formalism that corrects this mistake is presented. The new accurate formalism derives an accurate analysis of the X-ray reflectivity from a multilayer surface of thin film materials, taking into account the effect of roughness-induced diffuse scattering. The calculated reflectivity by this accurate reflectivity equation should enable the structure of buried interfaces to be analyzed more accurately.
\end{abstract}

\section{Introduction}

$\mathrm{X}$-rays scattered from a material surface at a glancing angle of incidence provide a wealth of information on the structure of the surface layer of materials. X-ray scattering spectroscopy is a powerful tool for investigations on rough surface and interface structures of multilayered thin film materials [123], and X-ray reflectometry is used for such investigations of various materials in many fields $[14,15,20]$. In many previous studies in X-ray reflectometry, the X-ray reflectivity was calculated based on the Parratt formalism [1], coupled with the use of the theory of Nevot and Croce to include roughness [2]. However, the calculated results of the X-ray reflectivity done in this way often showed strange results where the amplitude of the oscillation due to the interference effects would increase for a rougher surface.

Because the X-ray scattering vector in a specular reflectivity measurement is normal to the surface, it providesthe density profile solely in the direction perpendicular to surface. Specular reflectivity measurements can yield the magnitude of the average roughness perpendicular to surface and interfaces but cannot give information about the lateral extent of the roughness. In previous studies, the effect of roughness on the calculation of the X-ray reflectivity only took into account the effect of the density changes of the medium in a direction normal to the surface and interface. On the other hand, diffuse scattering can provide information about the lateral extent of the roughness. In contrast to previous calculations of the X-ray reflectivity, in the present analysis, we consider the effect of a decrease in the intensity of penetrated X-rays due to diffuse scattering at a rough surface and rough interface.

In this review, we show that the strange result has its origin in a currently used equation due to a serious mistake in which the Fresnel transmission coefficient in the reflectivity equation is increased at a rough interface, and the increase in the transmission coefficient completely overpowers any decrease in the value of the reflection coefficient because of 


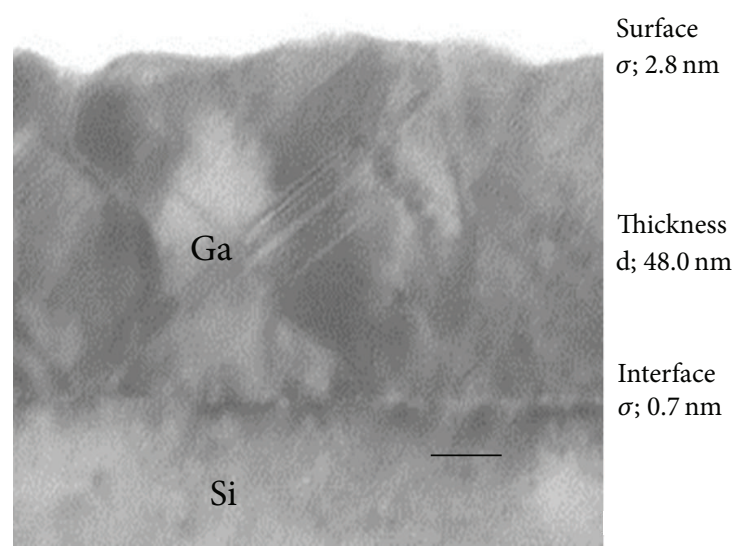

FIgure 1: Cross-section image of GaAs/Si(110) by TEM observation.

a lack of consideration of diffuse scattering. The mistake in Nevot and Croce's treatment originates in the fact that the modified Fresnel coefficients were calculated based on the theory which contains the X-ray energy conservation rule at surface and interface. In their discussion, the transmission coefficients were replaced approximately by the reflection coefficients by the ignoring diffuse scattering term at the rough interface and according to the principle of conservation energy at the rough interface also. The errors of transmittance without the modification cannot be ignored. It is meaningless to try to precisely match the numerical result based on a wrong calculating formula even to details of the reflectivity profile of the experimental result. Thus, because Nevot and Croce's treatment of the Parratt formalism contains a fundamental mistake regardless of the size of roughness, this approach needs to be corrected. In the present study, we present a new accurate formalism that corrects this mistake and thereby derive an accurate analysis of the X-ray reflectivity from a multilayer surface, taking into account the effect of roughness-induced diffuse scattering. The calculated reflectivity obtained by the use of this accurate reflectivity equation gives a physically reasonable result and should enable the structure of buried interfaces to be analyzed more accurately. This paper is the review article that is edited based on the two research articles of IOP Science $[22,23]$ and the later study.

\section{TEM Observation and X-Ray Reflectivity Measurement for Surfaces and Interfaces of Multilayered Thin Film Materials}

The surface and interfacial roughness of the same sample of multilayered thin film material was measured by transmission electron microscopy (TEM) and compared them with those from $\mathrm{X}$-ray reflectivity measurements. The surface sample for examination was prepared as follows; a GaAs layer was grown on $\mathrm{Si}(110)$ by molecular beam epitaxy (MBE). From TEM observations, the thickness of the GaAs layer was $48 \mathrm{~nm}$, the root-mean-square ( $\mathrm{rms}$ ) roughness of the GaAs surface was about $2.8 \mathrm{~nm}$, and the rms roughness of the interface between GaAs and $\mathrm{Si}$ was about $0.7 \mathrm{~nm}$. Figure 1

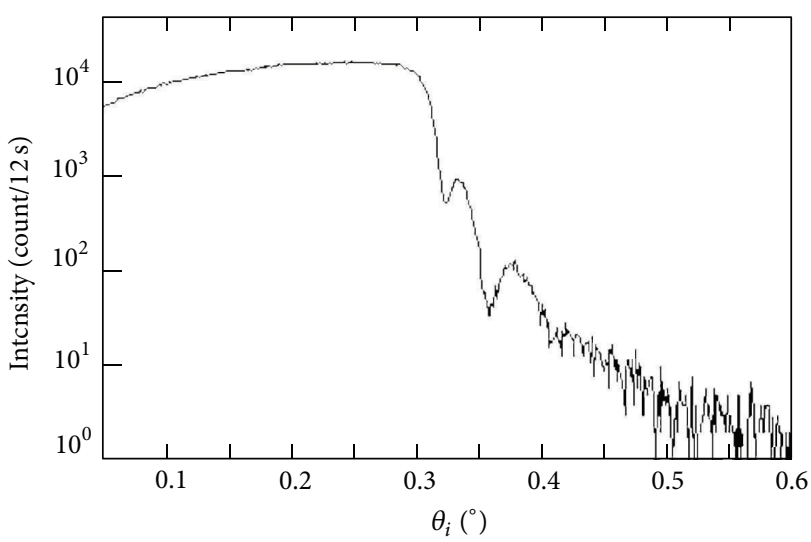

FIGURE 2: Measured X-ray reflectivity from a silicon wafer covered with a thin $(48 \mathrm{~nm})$ GaAs layer.

shows a cross-section image of this GaAs/Si(110) sample observed by TEM.

$\mathrm{X}$-ray reflectivity measurements were performed using a $\mathrm{Cu}-\mathrm{K} \alpha 1 \mathrm{X}$-ray beam from an $18 \mathrm{~kW}$ rotating-anode source. Figure 2 shows the measured reflectivity of $\mathrm{X}$-rays (wave length $0.154 \mathrm{~nm}$ ) from a GaAs layer with a thickness of $48 \mathrm{~nm}$ on a silicon wafer. The decrease in signal for angles larger than the total reflection critical angle shows oscillations. These oscillations are caused by interference between X-rays that reflect from the surface of GaAs layer and those that reflect from the interface of the GaAs layer and Si substrate. The characteristics of these oscillations reflect the surface roughness and the interface roughness.

\section{X-Ray Reflectivity Analysis}

In Section 3.1, we consider the calculation of the X-ray reflectivity from a multilayer material by the Parratt formalism [1], and in Section 3.2, the calculation of the X-ray reflectivity when roughness exists in the surface and the interface is considered.

3.1. X-ray Reflectivity from a Multilayer Material with a Flat Surface and Flat Interface. The intensity of X-rays propagating in the surface layers of a material, that is, the electric and magnetic fields, can be obtained from Maxwell's equations [24]. The effects of the material on the X-ray intensity are characterized by a complex refractive index $n$, which varies with depth. We divide a material in which the density changes continuously with depth into $N$ layers with an index $j$. The complex refractive index of the $j$ th layer is $n_{j}$. The vacuum is denoted as $j=0$ and $n_{0}=1$. The thickness of the $j$ th layer is $h_{j}$, the thickness of the bottom layer being assumed to be infinite.

The reflectance of an $N$-layer multilayer system can be calculated using the recursive formalism given by Parratt [1]. In the following, we show in detail the process of obtaining Parratt's expression and, further, show that this expression requires conservation of energy at the interface. We go on to 
show that the dispersion of the energy by interface roughness cannot be correctly accounted for Parratt's expression.

Following that approach, let $n_{j}$ be the refractive index of the $j$ th layer, defined as

$$
n_{j}=1-\delta_{j}-i \beta_{j},
$$

where $\delta_{j}$ and $\beta_{j}$ are the real and imaginary parts of the refractive index. These optical constants are related to the atomic scattering factor and electron density of the $j$ th layer material.

For X-rays of wavelength $\lambda$, the optical constants of the $j$ th layer material consisting of $N_{i j}$ atoms per unit volume can be expressed as

$$
\delta_{j}=\frac{\lambda^{2} r_{e}}{2 \pi} \sum_{i} f_{1 i} N_{i j}, \quad \beta_{j}=\frac{\lambda^{2} r_{e}}{2 \pi} \sum_{i} f_{2 i} N_{i j},
$$

where $r_{e}$ is the classical electron radius and $f_{1 i}$ and $f_{2 i}$ are the real and imaginary parts of the atomic scattering factor of the $i$ th element atom, respectively.

We take the vertical direction to the surface as the $z$-axis, with the positive direction pointing towards the bulk. The scattering plane is made the $x$-z-plane. The wave vector $\mathbf{k}_{j}$ of the $j$ th layer is related to the refractive index $n_{j}$ of the $j$ th layer by

$$
\frac{\mathbf{k}_{j} \cdot \mathbf{k}_{j}}{n_{j}^{2}}=\frac{\omega^{2}}{c^{2}}=\text { const },
$$

and, as this necessitates that the $x, y$-direction components of the wave vector are constant, then the $z$-direction component of the wave vector of the $j$ th layer is

$$
k_{j, z}=\sqrt{n_{j}^{2} \mathbf{k}_{0} \cdot \mathbf{k}_{0}-k_{0, x}^{2}} .
$$

In the 0th layer, that is, in vacuum,

$$
\begin{gathered}
n_{0}=1, \quad \mathbf{k}_{0} \cdot \mathbf{k}_{0}=k^{2}, \\
k=\frac{2 \pi}{\lambda}=\frac{\omega}{c} .
\end{gathered}
$$

In the $j$ th layer, the components of the wave vector are

$$
\begin{gathered}
k_{j, x}=k \cos \theta, \quad k_{j, y}=0, \\
k_{j, z}=k \sqrt{n_{j}^{2}-\cos ^{2} \theta} .
\end{gathered}
$$

The electric field of X-ray radiation at a glancing angle of incidence $\theta$ is expressed as

$$
\mathbf{E}_{0}(z)=\mathbf{A}_{0} \exp \left[i\left(\mathbf{k}_{0} \cdot \mathbf{r}-\omega t\right)\right] .
$$

The incident radiation is usually decomposed into two geometries to simplify the analysis, one with the incident electric field $E$ parallel to the plane of incidence ( $p$-polarization) and one with $E$ perpendicular to that plane (s-polarization). An arbitrary incident wave can be represented in terms of these two polarizations. Thus, $E_{0 x}$ and $E_{0 z}$ correspond to

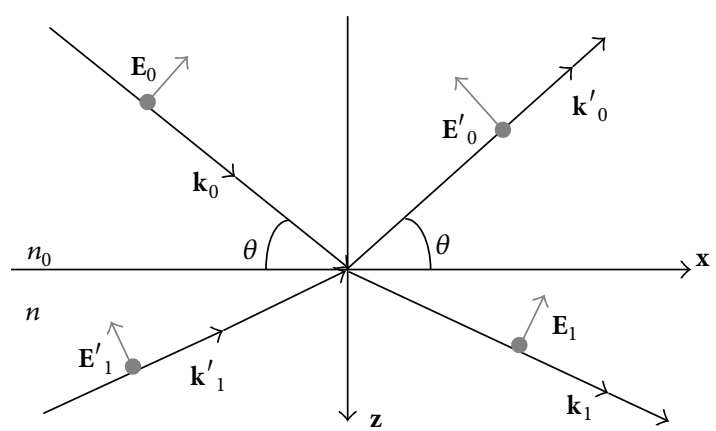

Figure 3: Reflected and transmitted X-rays.

$p$-polarization and $E_{0 y}$ to $s$-polarization; those components of the amplitude's electric vector are expressed as

$$
\begin{gathered}
A_{0 x}=-A_{0 p} \sin \theta, \quad A_{0 y}=A_{0 s}, \\
A_{0 z}=A_{0 p} \cos \theta .
\end{gathered}
$$

The components of the wave vector of the incident X-rays are

$$
k_{0 x}=k \cos \theta, \quad k_{0 y}=0, \quad k_{0 z}=k \sin \theta .
$$

The electric field of reflected X-ray radiation of exit angle $\theta$ is expressed as

$$
\mathbf{E}_{0}^{\prime}(z)=\mathbf{A}_{0}^{\prime} \exp \left[i\left(\mathbf{k}_{0}^{\prime} \cdot \mathbf{r}-\omega t\right)\right]
$$

where

$$
k_{0 x}^{\prime}=k_{0 x}, \quad k_{0 y}^{\prime}=0, \quad k_{0 z}^{\prime}=-k_{0 z} .
$$

Because an X-ray is a transverse wave, the amplitude and the wave vector are orthogonal as follows:

$$
\mathbf{A}_{j} \cdot \mathbf{k}_{j}=0, \quad \mathbf{A}_{j}^{\prime} \cdot \mathbf{k}_{j}^{\prime}=0 .
$$

We consider the relation of the electric field $\mathbf{E}_{0}$ of X-rays incident at a flat surface from vacuum, the electric field $\mathbf{E}_{1}$ of X-rays propagating in the first layer material, the electric field $\mathbf{E}_{0}^{\prime}$ of X-rays reflected from the surface exit to vacuum, and the electric field $\mathbf{E}_{1}^{\prime}$ of $\mathrm{X}$-rays propagating toward to the surface in the first layer material, as shown in Figure 3.

The electric fields $\mathbf{E}_{1}, \mathbf{E}_{1}^{\prime}$ in the first layer material below the surface are expressed as

$$
\begin{aligned}
& \mathbf{E}_{1}(z)=\mathbf{A}_{1} \exp \left[i\left(\mathbf{k}_{1} \cdot \mathbf{r}-\omega t\right)\right], \\
& \mathbf{E}_{1}^{\prime}(z)=\mathbf{A}_{1}^{\prime} \exp \left[i\left(\mathbf{k}_{1}^{\prime} \cdot \mathbf{r}-\omega t\right)\right] .
\end{aligned}
$$

where

$$
\begin{gathered}
k_{1 x}^{\prime}=k_{1 x}, \quad k_{1 y}^{\prime}=0, \quad k_{1 z}^{\prime}=-k_{1 z}, \\
k_{1, x}=k \cos \theta, \quad k_{1 y}=0, \quad k_{1, z}=k \sqrt{n_{1}^{2}-\cos ^{2} \theta} .
\end{gathered}
$$


The relation of the amplitudes $A_{0}, A_{0}^{\prime}, A_{1}$, and $A_{1}^{\prime}$ can be found from the continuity equations of the electric fields for the interface between the 0th and 1th layers as follows:

$$
\begin{gathered}
A_{0, x}+A_{0, x}^{\prime}=A_{1, x}+A_{1, x}^{\prime}, \\
A_{0, y}+A_{0, y}^{\prime}=A_{1, y}+A_{1, y}^{\prime}, \\
k_{0, x} A_{0, x}+k_{0, x}^{\prime} A_{0, x}^{\prime}=k_{1, x} A_{1, x}+k_{1, x}^{\prime} A_{1, x}^{\prime}, \\
k_{0, y} A_{0, y}+k_{0, y}^{\prime} A_{0, y}^{\prime}=k_{1, y} A_{1, y}+k_{1, y}^{\prime} A_{1, y}^{\prime} .
\end{gathered}
$$

Another relation of the amplitudes $A_{0}, A_{0}^{\prime}, A_{1}$, and $A_{1}^{\prime}$ can be found from the continuity equations of the magnetic fields for the interface between the 0th and 1th layers is shown as follows:

$$
\begin{aligned}
& k_{0, z} A_{0, y}-k_{0, y} A_{0, z}+k_{0, z}^{\prime} A_{0, y}^{\prime}-k_{0, y}^{\prime} A_{0, z}^{\prime} \\
& =k_{1, z} A_{1, y}-k_{1, y} A_{1, z}+k_{1, z}^{\prime} A_{1, y}^{\prime}-k_{1, y}^{\prime} A_{1, z}^{\prime}, \\
& k_{0, z} A_{0, x}-k_{0, x} A_{0, z}+k_{0, z}^{\prime} A_{0, x}^{\prime}-k_{0, x}^{\prime} A_{0, z}^{\prime} \\
& =k_{1, z} A_{1, x}-k_{1, x} A_{1, z}+k_{1, z}^{\prime} A_{1, x}^{\prime}-k_{1, x}^{\prime} A_{1, z}^{\prime} .
\end{aligned}
$$

From the previous equations, these amplitudes are related by the Fresnel coefficient tensor $\boldsymbol{\Phi}$ for refraction and the Fresnel coefficient tensor $\Psi$ for reflection as follows:

$$
\left(\begin{array}{l}
\mathbf{A}_{0}^{\prime} \\
\mathbf{A}_{1}
\end{array}\right)=\left(\begin{array}{ll}
\Psi_{0,1} & \boldsymbol{\Phi}_{1,0} \\
\boldsymbol{\Phi}_{0,1} & \boldsymbol{\Psi}_{1,0}
\end{array}\right)\left(\begin{array}{l}
\mathbf{A}_{0} \\
\mathbf{A}_{1}^{\prime}
\end{array}\right) .
$$

Here, the Fresnel coefficient tensor $\boldsymbol{\Phi}$ for refraction at the interface between the 0 th and 1th layers is given by

$$
\begin{aligned}
& \Phi_{0,1, x x}=\frac{2 k_{1, z} \mathbf{k}_{0} \cdot \mathbf{k}_{0}}{k_{0, z} \mathbf{k}_{1} \cdot \mathbf{k}_{1}+k_{1, z} \mathbf{k}_{0} \cdot \mathbf{k}_{0}}, \\
& \Phi_{1,0, x x}=\frac{2 k_{0, z} \mathbf{k}_{1} \cdot \mathbf{k}_{1}}{k_{0, z} \mathbf{k}_{1} \cdot \mathbf{k}_{1}+k_{1, z} \mathbf{k}_{0} \cdot \mathbf{k}_{0}}, \\
& \Phi_{0,1, y y}=\frac{2 k_{0, z}}{k_{0, z}+k_{1, z}}, \\
& \Phi_{1,0, y y}=\frac{2 k_{1, z}}{k_{1, z}+k_{0, z}}, \\
& \Phi_{0,1, z z}=\frac{2 k_{0, z} \mathbf{k}_{0} \cdot \mathbf{k}_{0}}{k_{0, z} \mathbf{k}_{1} \cdot \mathbf{k}_{1}+k_{1, z} \mathbf{k}_{0} \cdot \mathbf{k}_{0}}, \\
& \Phi_{1,0, z z}=\frac{2 k_{1, z} \mathbf{k}_{1} \cdot \mathbf{k}_{1}}{k_{0, z} \mathbf{k}_{1} \cdot \mathbf{k}_{1}+k_{1, z} \mathbf{k}_{0} \cdot \mathbf{k}_{0}}, \\
& \Phi_{0,1, x y}=\Phi_{0,1, y x}=\Phi_{0,1, y z}=0, \\
& \Phi_{1,0, x y}=\Phi_{1,0, y x}=\Phi_{1,0, y z}=0, \\
& \Phi_{0,1, z y}=\Phi_{0,1, z x}=\Phi_{0,1, x z}=0, \\
& \Phi_{1,0, z y}=\Phi_{1,0, z x}=\Phi_{1,0, x z}=0 .
\end{aligned}
$$

The Fresnel coefficient tensor $\boldsymbol{\Psi}$ for reflection from the interface between the 0th and 1th layers is given by

$$
\begin{aligned}
& \Psi_{0,1, x x}=\frac{k_{1, z} \mathbf{k}_{0} \cdot \mathbf{k}_{0}-k_{0, z} \mathbf{k}_{1} \cdot \mathbf{k}_{1}}{k_{0, z} \mathbf{k}_{1} \cdot \mathbf{k}_{1}+k_{1, z} \mathbf{k}_{0} \cdot \mathbf{k}_{0}}, \\
& \Psi_{1,0, x x}=\frac{k_{0, z} \mathbf{k}_{1} \cdot \mathbf{k}_{1}-k_{1, z} \mathbf{k}_{0} \cdot \mathbf{k}_{0}}{k_{0, z} \mathbf{k}_{1} \cdot \mathbf{k}_{1}+k_{1, z} \mathbf{k}_{0} \cdot \mathbf{k}_{0}}, \\
& \Psi_{0,1, y y}=\frac{k_{0, z}-k_{1, z}}{k_{0, z}+k_{1, z}}, \\
& \Psi_{1,0, y y}=\frac{k_{1, z}-k_{0, z}}{k_{0, z}+k_{1, z}}, \\
& \Psi_{0,1, z z}=-\frac{k_{1, z} \mathbf{k}_{0} \cdot \mathbf{k}_{0}-k_{0, z} \mathbf{k}_{1} \cdot \mathbf{k}_{1}}{k_{0, z} \mathbf{k}_{1} \cdot \mathbf{k}_{1}+k_{1, z} \mathbf{k}_{0} \cdot \mathbf{k}_{0}}, \\
& \Psi_{1,0, z z}=\frac{k_{1, z} \mathbf{k}_{0} \cdot \mathbf{k}_{0}-k_{0, z} \mathbf{k}_{1} \cdot \mathbf{k}_{1}}{k_{0, z} \mathbf{k}_{1} \cdot \mathbf{k}_{1}+k_{1, z} \mathbf{k}_{0} \cdot \mathbf{k}_{0}}, \\
& \Psi_{0,1, x y}=\Psi_{0,1, y x}=\Psi_{0,1, y z}=0, \\
& \Psi_{1,0, x y}=\Psi_{1,0, y x}=\Psi_{1,0, y z}=0, \\
& \Psi_{0,1, z y}=\Psi_{0,1, z x}=\Psi_{0,1, x z}=0, \\
& \Psi_{1,0, z x}=\Psi_{1,0, x z}=0 .
\end{aligned}
$$

Here, we consider the reflection from a flat surface of a single layer. The reflection coefficient is defined as the ratio $R_{0,1}$ of the reflected electric field to the incident electric field at the surface of the material. The reflection coefficient $R_{0,1}$ from a single-layer flat surface is equal to the Fresnel coefficient $\Psi_{0,1}$ for reflection, as the following shows

$$
\mathbf{A}_{0}^{\prime}=\mathbf{R}_{0,1} \mathbf{A}_{0}=\Psi_{0,1} \mathbf{A}_{0}
$$

In general, when X-rays that are linearly polarized at an angle $\chi$ impinge on the surface at an angle of incidence $\theta$, the components of the amplitude's electric vector are expressed as

$$
\begin{gathered}
\mathbf{A}_{0}=\left(\begin{array}{c}
A_{0 x} \\
A_{0 y} \\
A_{0 z}
\end{array}\right)=\left(\begin{array}{c}
-A_{0 p} \sin \theta \\
A_{0 s} \\
A_{0 p} \cos \theta
\end{array}\right), \\
\left(\begin{array}{c}
A_{0 p} \\
A_{0 s}
\end{array}\right)=\left(\begin{array}{c}
A_{0} \sin \chi \\
A_{0} \cos \chi
\end{array}\right) .
\end{gathered}
$$


The amplitudes of reflected X-ray radiation are expressed as

$$
\begin{aligned}
& \mathbf{A}_{0}^{\prime}=\left(\begin{array}{c}
A_{0 x}^{\prime} \\
A_{0 y}^{\prime} \\
A_{0 z}^{\prime}
\end{array}\right) \\
& =\left(\begin{array}{ccc}
\Psi_{0,1, x x} & 0 & 0 \\
0 & \Psi_{0,1, y y} & 0 \\
0 & 0 & \Psi_{0,1, z z}
\end{array}\right)\left(\begin{array}{l}
A_{0 x} \\
A_{0 y} \\
A_{0 z}
\end{array}\right), \\
& \mathbf{A}_{0}^{\prime}=\left(\begin{array}{c}
A_{0 x}^{\prime} \\
A_{0 y}^{\prime} \\
A_{0 z}^{\prime}
\end{array}\right)=A_{0}\left(\begin{array}{c}
-\Psi_{0,1, x x} \sin \chi \sin \theta \\
\Psi_{0,1, y y} \cos \chi \\
\Psi_{0,1, z z} \sin \chi \cos \theta
\end{array}\right) \text {. }
\end{aligned}
$$

The X-ray reflectivity $R$ is

$$
R=\left|\mathbf{R}_{0,1}\right|=\frac{\left|\mathbf{A}_{0}^{\prime} \cdot \mathbf{A}_{0}^{\prime}\right|}{\left|\mathbf{A}_{0} \cdot \mathbf{A}_{0}\right|} .
$$

Then

$$
\begin{aligned}
R= & \Psi_{0,1, x x} \Psi_{0,1, x x}^{*} \sin ^{2} \chi \sin ^{2} \theta \\
& +\Psi_{0,1, y y} \Psi_{0,1, y y}^{*} \cos ^{2} \chi+\Psi_{0,1, z z} \Psi_{0,1, z z}^{*} \sin ^{2} \chi \cos ^{2} \theta,
\end{aligned}
$$

where

$$
\begin{aligned}
& \Psi_{0,1, x x}=\frac{k_{1, z} n_{0}^{2}-k_{0, z} n_{1}^{2}}{k_{0, z} n_{1}^{2}+k_{1, z} n_{0}^{2}}, \\
& \Psi_{0,1, y y}=\frac{k_{0, z}-k_{1, z}}{k_{0, z}+k_{1, z}}, \\
& \Psi_{0,1, z z}=-\frac{k_{1, z} n_{0}^{2}-k_{0, z} n_{1}^{2}}{k_{0, z} n_{1}^{2}+k_{1, z} n_{0}^{2}}, \\
& \Psi_{0,1, x x} \Psi_{0,1, x x}^{*}=\frac{-k_{0, z} n_{1}^{2}+k_{1, z}}{k_{0, z} n_{1}^{2}+k_{1, z}} \frac{k_{0, z} n_{1}^{* 2}+k_{1, z}^{*}}{k_{0, z} n_{1}^{* 2}+k_{1, z}^{*}}, \\
& \Psi_{0,1, y y} \Psi_{0,1, y y}^{*}=\frac{k_{0, z}-k_{1, z}}{k_{0, z}+k_{1, z}} \frac{k_{0, z}-k_{1, z}^{*}}{k_{0, z}+k_{1, z}^{*}}, \\
& \Psi_{0,1, z z} \Psi_{0,1, z z}^{*}=\frac{k_{0, z} n_{1}^{2}-k_{1, z}}{k_{0, z} n_{1}^{2}+k_{1, z}} \frac{k_{0, z} n_{1}^{* 2}-k_{1, z}^{*}}{k_{0, z} n_{1}^{* 2}+k_{1, z}^{*}}, \\
& =\Psi_{0,1, x x} \Psi_{0,1, x x}^{*} .
\end{aligned}
$$

Then,

$$
R=\Psi_{0,1, y y} \Psi_{0,1, y y}^{*} \cos ^{2} \chi+\Psi_{0,1, z z} \Psi_{0,1, z z}^{*} \sin ^{2} \chi .
$$

Taking an average for $\chi$,

$$
R=\left\langle\Psi_{0,1, y y} \Psi_{0,1, y y}^{*} \cos ^{2} \chi+\Psi_{0,1, z z} \Psi_{0,1, z z}^{*} \sin ^{2} \chi\right\rangle_{\chi}
$$

Then

$$
R=\frac{\left(\Psi_{0,1, y y} \Psi_{0,1, y y}^{*}+\Psi_{0,1, z z} \Psi_{0,1, z z}^{*}\right)}{2} .
$$

For the reflectivity in the case of $s$-polarized X-rays incident,

$$
R=\Psi_{0,1, y y} \Psi_{0,1, y y}^{*} .
$$

Next, we consider the reflection from a flat surface of a multilayer with flat interfaces. We consider the electric field $\mathbf{E}_{j-1}$ of X-rays propagating in the $(j-1)$ th layer material, the electric field $\mathbf{E}_{j}$ of $\mathrm{X}$-rays propagating in the $j$ th layer material, and the electric field $\mathbf{E}_{j-1}^{\prime}$ of X-rays reflected from the $j$ th layer material at $z=z_{j-1, j}$ of the interface between the $(j-1)$ th layer and $j$ th layers as shown in Figure 4.

The electric fields $\mathbf{E}_{j-1}, \mathbf{E}_{j-1}^{\prime}$ at the interface between the $(j-1)$ th layer and $j$ th layer and the electric fields $\mathbf{E}_{j}, \mathbf{E}_{j}^{\prime}$ below the interface between the $(j-1)$ th layer and $j$ th layer are expressed as

$$
\begin{aligned}
& \mathbf{E}_{j-1}\left(z_{j-1, j}\right) \\
& =\mathbf{A}_{j-1} \exp \left[i\left(k_{j-1, x} x+k_{j-1, y} y+k_{j-1, z} h_{j-1}-\omega t\right)\right], \\
& \mathbf{E}_{j-1}^{\prime}\left(z_{j-1, j}\right) \\
& =\mathbf{A}_{j-1}^{\prime} \exp \left[i\left(k_{j-1, x} x+k_{j-1, y} y-k_{j-1, z} h_{j-1}-\omega t\right)\right], \\
& \quad \mathbf{E}_{j}\left(z_{j-1, j}\right)=\mathbf{A}_{j} \exp \left[i\left(k_{j, x} x+k_{j, y} y-\omega t\right)\right], \\
& \quad \mathbf{E}_{j}^{\prime}\left(z_{j-1, j}\right)=\mathbf{A}_{j}^{\prime} \exp \left[i\left(k_{j, x} x+k_{j, y} y-\omega t\right)\right] .
\end{aligned}
$$

The electric fields of X-rays at the interface between the $(j-$ $1)$ th layer and $j$ th layer can be formally expressed as follows:

$$
\begin{array}{r}
\mathbf{E}_{j}\left(z_{j-1, j}\right)=\boldsymbol{\Phi}_{j-1, j} \mathbf{E}_{j-1}\left(z_{j-1, j}\right)+\Psi_{j, j-1} \mathbf{E}_{j}^{\prime}\left(z_{j-1, j}\right), \\
\mathbf{E}_{j-1}^{\prime}\left(z_{j-1, j}\right)=\Psi_{j-1, j} \mathbf{E}_{j-1}\left(z_{j-1, j}\right)+\boldsymbol{\Phi}_{j, j-1} \mathbf{E}_{j}^{\prime}\left(z_{j-1, j}\right),
\end{array}
$$

where $\Psi_{j-1, j}$ is the Fresnel coefficient tensor for reflection from the interface between the $j-1$ and $j$ layers, and $\Phi_{j-1, j}$ is the Fresnel coefficient tensor for refraction at the interface between the $j-1$ and $j$ layers. In addition, the electric field within the $j$ th layer varies with depth $h_{j}$ as follows:

$$
\begin{aligned}
\mathbf{E}_{j}\left(z_{j, j+1}\right) & =\mathbf{E}_{j}\left(z_{j-1, j}\right) \exp \left(i k_{j, z} h_{j}\right), \\
\mathbf{E}_{j}^{\prime}\left(z_{j, j+1}\right) & =\mathbf{E}_{j}^{\prime}\left(z_{j-1, j}\right) \exp \left(-i k_{j, z} h_{j}\right) .
\end{aligned}
$$

The amplitudes $A_{j}$ and $A_{j}^{\prime}$ at the $j$ th layer are derived from the previous equations for the interface between the $j-1$ and $j$ layers as follows:

$$
\begin{aligned}
& \mathbf{A}_{j-1}^{\prime} \exp \left(-i k_{j-1, z} h_{j-1}\right) \\
& \quad=\boldsymbol{\Psi}_{j-1, j} \mathbf{A}_{j-1} \exp \left(i k_{j-1, z} h_{j-1}\right)+\boldsymbol{\Phi}_{j, j-1} \mathbf{A}_{j}^{\prime}, \\
& \mathbf{A}_{j}=\boldsymbol{\Phi}_{j-1, j} \mathbf{A}_{j-1} \exp \left(i k_{j-1, z} h_{j-1}\right)+\boldsymbol{\Psi}_{j, j-1} \mathbf{A}_{j}^{\prime} .
\end{aligned}
$$




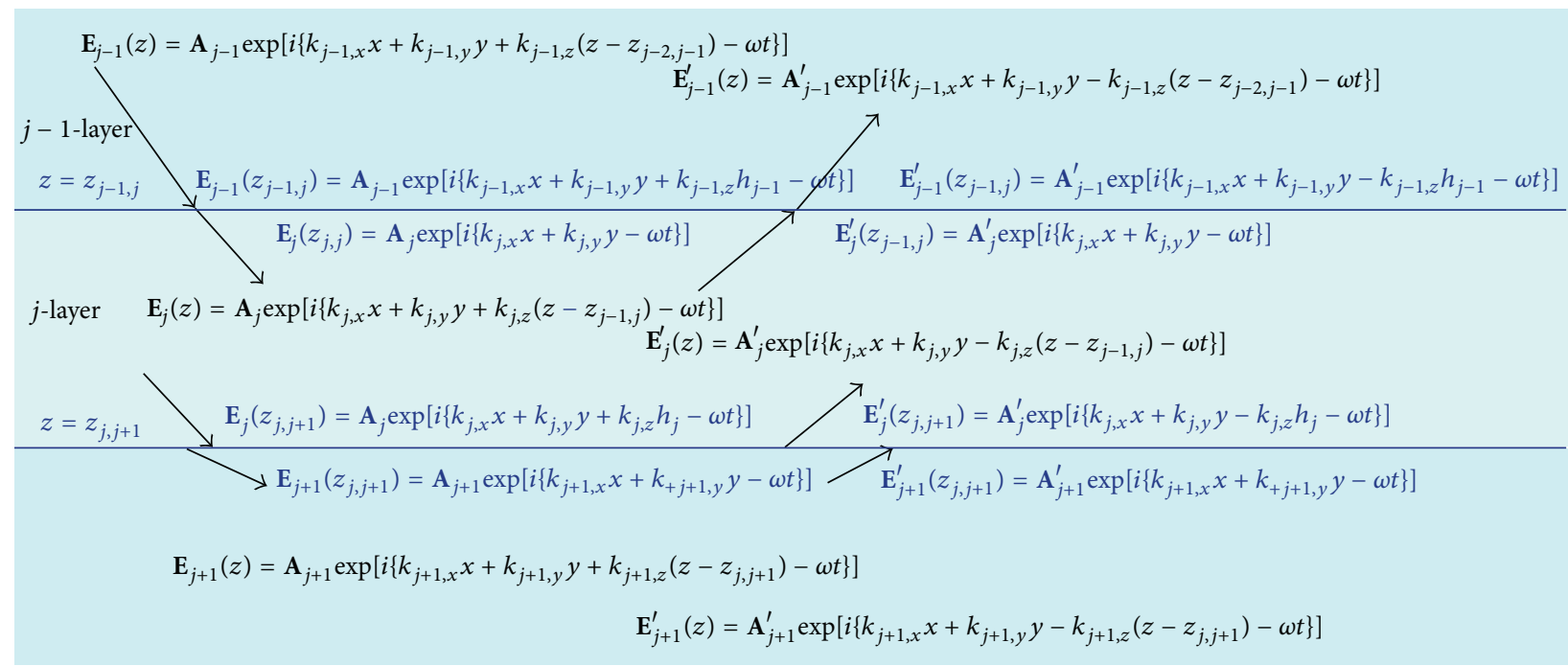

FIgURE 4: Reflection and transmission of X-rays in the $(j-1)$ th, $j$ th, and $(j+1)$ th layers of a multilayer material.

This relation is expressed by the following matrix:

$$
\begin{aligned}
& \left(\begin{array}{c}
\mathbf{A}_{j-1}^{\prime} \exp \left(-i k_{j-1, z} h_{j-1}\right) \\
\mathbf{A}_{j}
\end{array}\right) \\
& \quad=\left(\begin{array}{cc}
\boldsymbol{\Psi}_{j-1, j} & \boldsymbol{\Phi}_{j, j-1} \\
\boldsymbol{\Phi}_{j-1, j} & \boldsymbol{\Psi}_{j, j-1}
\end{array}\right)\left(\begin{array}{c}
\mathbf{A}_{j-1} \exp \left(i k_{j-1, z} h_{j-1}\right) \\
\mathbf{A}_{j}^{\prime}
\end{array}\right) .
\end{aligned}
$$

Here, the Fresnel coefficient tensor $\Phi$ for refraction at the interface between the $(j-1)$ th and $j$ th layers is given by

$$
\begin{aligned}
\Phi_{j-1, j, x x} & =\frac{2 k_{j, z} \mathbf{k}_{j-1} \cdot \mathbf{k}_{j-1}}{k_{j-1, z} \mathbf{k}_{j} \cdot \mathbf{k}_{j}+k_{j, z} \mathbf{k}_{j-1} \cdot \mathbf{k}_{j-1}}, \\
\Phi_{j, j-1, x x} & =\frac{2 k_{j-1, z} \mathbf{k}_{j} \cdot \mathbf{k}_{j}}{k_{j-1, z} \mathbf{k}_{j} \cdot \mathbf{k}_{j}+k_{j, z} \mathbf{k}_{j-1} \cdot \mathbf{k}_{j-1}}, \\
\Phi_{j-1, j, y y} & =\frac{2 k_{j-1, z}}{k_{j-1, z}+k_{j, z}}, \\
\Phi_{j, j-1, y y} & =\frac{2 k_{j, z}}{k_{j, z}+k_{j-1, z}}, \\
\Phi_{j-1, j, z z} & =\frac{2 k_{j-1, z} \mathbf{k}_{j-1} \cdot \mathbf{k}_{j-1}}{k_{j-1, z} \mathbf{k}_{j} \cdot \mathbf{k}_{j}+k_{j, z} \mathbf{k}_{j-1} \cdot \mathbf{k}_{j-1}} \\
\Phi_{j, j-1, z z} & =\frac{2 k_{j, z} \mathbf{k}_{j} \cdot \mathbf{k}_{j}}{k_{j-1, z} \mathbf{k}_{j} \cdot \mathbf{k}_{j}+k_{j, z} \mathbf{k}_{j-1} \cdot \mathbf{k}_{j-1}} \\
\Phi_{j-1, j, x y} & =\Phi_{j-1, j, y x}=\Phi_{j-1, j, y z}=0, \\
\Phi_{j, j-1, x y} & =\Phi_{j, j-1, y x}=\Phi_{j, j-1, y z}=0 \\
\Phi_{j-1, j, z y} & =\Phi_{j-1, j, z x}=\Phi_{j-1, j, x z}=0, \\
\Phi_{j, j-1, z y} & =\Phi_{j, j-1, z x}=\Phi_{j, j-1, x z}=0 .
\end{aligned}
$$

The Fresnel coefficient tensor $\Psi$ for reflection from the interface between the $j-1$ and $j$ layers is given by

$$
\begin{aligned}
& \Psi_{j-1, j, x x}=\frac{k_{j, z} \mathbf{k}_{j-1} \cdot \mathbf{k}_{j-1}-k_{j-1, z} \mathbf{k}_{j} \cdot \mathbf{k}_{j}}{k_{j-1, z} \mathbf{k}_{j} \cdot \mathbf{k}_{j}+k_{j, z} \mathbf{k}_{j-1} \cdot \mathbf{k}_{j-1}}, \\
& \Psi_{j, j-1, x x}=\frac{k_{j-1, z} \mathbf{k}_{j} \cdot \mathbf{k}_{j}-k_{j, z} \mathbf{k}_{j-1} \cdot \mathbf{k}_{j-1}}{k_{j-1, z} \mathbf{k}_{j} \cdot \mathbf{k}_{j}+k_{j, z} \mathbf{k}_{j-1} \cdot \mathbf{k}_{j-1}}, \\
& \Psi_{j-1, j, y y}=\frac{k_{j-1, z}-k_{j, z}}{k_{j-1, z}+k_{j, z}} \\
& \Psi_{j, j-1, y y}=\frac{k_{j, z}-k_{j-1, z}}{k_{j-1, z}+k_{j, z}}, \\
& \Psi_{j-1, j, z z}=-\frac{k_{j, z} \mathbf{k}_{j-1} \cdot \mathbf{k}_{j-1}-k_{j-1, z} \mathbf{k}_{j} \cdot \mathbf{k}_{j}}{k_{j-1, z} \mathbf{k}_{j} \cdot \mathbf{k}_{j}+k_{j, z} \mathbf{k}_{j-1} \cdot \mathbf{k}_{j-1}}, \\
& \Psi_{j, j-1, z z}=\frac{k_{j, z} \mathbf{k}_{j-1} \cdot \mathbf{k}_{j-1}-k_{j-1, z} \mathbf{k}_{j} \cdot \mathbf{k}_{j}}{k_{j-1, z} \mathbf{k}_{j} \cdot \mathbf{k}_{j}+k_{j, z} \mathbf{k}_{j-1} \cdot \mathbf{k}_{j-1}}, \\
& \Psi_{j-1, j, x y}=\Psi_{j-1, j, y x}=\Psi_{j-1, j, y z}=0, \\
& \Psi_{j, j-1, x y}=\Psi_{j, j-1, y x}=\Psi_{j, j-1, y z}=0, \\
& \Psi_{j-1, j, z y}=\Psi_{j-1, j, z x}=\Psi_{j-1, j, x z}=0, \\
& \Psi_{j, j-1, z y}=\Psi_{j, j-1, z x}=\Psi_{j, j-1, x z}=0 .
\end{aligned}
$$

The amplitudes $\mathbf{A}_{j-1}$ and $\mathbf{A}_{j-1}^{\prime}$ of the electric fields $\mathbf{E}_{j-1}$, $\mathbf{E}_{j-1}^{\prime}$ at the $j$ th layer and the amplitudes $\mathbf{A}_{j}$ and $\mathbf{A}_{j}^{\prime}$ of 
the electric fields $\mathbf{E}_{j}, \mathbf{E}_{j}^{\prime}$ at the $(j+1)$ th layer are related by the following equations:

$$
\begin{aligned}
& \left(\begin{array}{cc}
\boldsymbol{\Phi}_{j-1, j} & 0 \\
0 & \boldsymbol{\Phi}_{j-1, j}
\end{array}\right)\left(\begin{array}{c}
\mathbf{A}_{j-1} \\
\mathbf{A}_{j-1}^{\prime}
\end{array}\right) \\
& =\left(\begin{array}{cc}
\exp \left(-i k_{j-1, z} h_{j-1}\right) & 0 \\
0 & \exp \left(i k_{j-1, z} h_{j-1}\right)
\end{array}\right) \\
& \quad \times\left(\begin{array}{cc}
1 & -\boldsymbol{\Psi}_{j, j-1} \\
\boldsymbol{\Psi}_{j-1, j} & \left(\boldsymbol{\Phi}_{j-1, j} \boldsymbol{\Phi}_{j, j-1}-\boldsymbol{\Psi}_{j-1, j} \boldsymbol{\Psi}_{j, j-1}\right)
\end{array}\right)\left(\begin{array}{c}
\mathbf{A}_{j} \\
\mathbf{A}_{j}^{\prime}
\end{array}\right) .
\end{aligned}
$$

For $s$-polarization, the Fresnel coefficients are

$$
\begin{array}{ll}
\Phi_{j-1, j, y y}=\frac{2 k_{j-1, z}}{k_{j-1, z}+k_{j, z}}, & \Phi_{j, j-1, y y}=\frac{2 k_{j, z}}{k_{j-1, z}+k_{j, z}} . \\
\Psi_{j-1, j, y y}=\frac{k_{j-1, z}-k_{j, z}}{k_{j-1, z}+k_{j, z}}, & \Psi_{j, j-1, y y}=\frac{k_{j, z}-k_{j-1, z}}{k_{j-1, z}+k_{j, z}} .
\end{array}
$$

Then, the relations between the amplitudes $A_{j-1}, A_{j-1}^{\prime}, A_{j}$, and $A_{j}^{\prime}$ at the interface of the $(j-1)$ th and $j$ th layers are expressed as follows:

$$
\begin{aligned}
\left(\begin{array}{c}
A_{j-1} \\
A_{j-1}^{\prime}
\end{array}\right)= & \left(\begin{array}{cc}
\exp \left(-i k_{j-1, z} h_{j-1}\right) & 0 \\
0 & \exp \left(i k_{j-1, z} h_{j-1}\right)
\end{array}\right) \\
& \times\left(\begin{array}{cc}
\frac{k_{j-1, z}+k_{j, z}}{2 k_{j-1, z}} & \frac{k_{j-1, z}-k_{j, z}}{2 k_{j-1, z}} \\
\frac{k_{j-1, z}-k_{j, z}}{2 k_{j-1, z}} & \frac{k_{j-1, z}+k_{j, z}}{2 k_{j-1, z}}
\end{array}\right)\left(\begin{array}{c}
A_{j} \\
A_{j}^{\prime}
\end{array}\right) .
\end{aligned}
$$

The reflection coefficient is defined as the ratio $\mathbf{R}_{0,1}$ of the reflected electric field to the incident electric field at the surface of the material and is given by

$$
\mathbf{A}_{0}^{\prime}=\mathbf{R}_{0,1} \mathbf{A}_{0} \text {. }
$$

The reflection coefficient $\mathbf{R}_{j-1, j}$ of the electric field $\mathbf{E}_{j-1}^{\prime}$ to the electric field $\mathbf{E}_{j-1}$ at the interface of $(j-1)$ th layer and $j$ th layer is

$$
\mathbf{A}_{j-1}^{\prime}=\mathbf{R}_{j-1, j} \mathbf{A}_{j-1},
$$

and the ratio $R_{j-1, j}$ is related to the ratio $R_{j, j+1}$ as follows:

$$
\begin{aligned}
\mathbf{R}_{j-1, j}= & \frac{\boldsymbol{\Psi}_{j-1, j}+\left(\boldsymbol{\Phi}_{j-1, j} \boldsymbol{\Phi}_{j, j-1}-\boldsymbol{\Psi}_{j-1, j} \boldsymbol{\Psi}_{j, j-1}\right) \mathbf{R}_{j, j+1}}{1-\boldsymbol{\Psi}_{j, j-1} \mathbf{R}_{j, j+1}} \\
& \times \exp \left(2 i k_{j-1, z} h_{j-1}\right) .
\end{aligned}
$$

Here, from the relation between the Fresnel coefficient for reflection and the Fresnel coefficient for refraction,

$$
\begin{gathered}
\boldsymbol{\Phi}_{j-1, j} \boldsymbol{\Phi}_{j, j-1}-\boldsymbol{\Psi}_{j-1, j} \boldsymbol{\Psi}_{j, j-1}=1, \\
\boldsymbol{\Psi}_{j-1, j}=-\boldsymbol{\Psi}_{j, j-1} .
\end{gathered}
$$

We can formulate the following relationship:

$$
\mathbf{R}_{j-1, j}=\frac{\boldsymbol{\Psi}_{j-1, j}+\mathbf{R}_{j, j+1}}{1+\boldsymbol{\Psi}_{j-1, j} \mathbf{R}_{j, j+1}} \exp \left(2 i k_{j-1, z} h_{j-1}\right) \text {. }
$$

It is reasonable to assume that no wave will be reflected back from the substrate, so that

$$
\mathbf{R}_{N, N+1}=0 \text {. }
$$

Then, the X-ray reflectivity is simply

$$
R=\left|R_{0,1}\right|^{2}
$$

3.2. Previous Calculations of X-Ray Reflectivity When Roughness Exists at the Surface and Interface. When the surface and interface have roughness, the Fresnel coefficient for reflection is reduced by the roughness [8-19]. The effect of the roughness was previously put into the calculation based on the theory of Nevot and Croce [2]. The effect of such roughness was taken into account only through the effect of the changes in density of the medium in a vertical direction to the surface and interface. With the use of relevant roughness parameters like the root-mean-square (rms) roughness $\sigma_{j-1, j}$ of the $j$ th layer, the reduced Fresnel reflection coefficient $\Psi^{\prime}$ for $s$-polarization is transformed as follows:

$$
\Psi_{j, j-1}^{\prime}=\Psi_{j, j-1} \exp \left(-2 k_{j, z} k_{j-1, z} \sigma_{j, j-1}^{2}\right),
$$

and the X-ray reflectivity is calculated using the following equation:

$$
R_{j-1, j}=\frac{R_{j, j+1}+\Psi_{j-1, j}^{\prime}}{1+R_{j, j+1} \Psi_{j-1, j}^{\prime}} \exp \left(2 i k_{j-1, z} h_{j-1}\right) .
$$

Figure 5 shows the result (dots) of a calculation based on these expressions of the reflectivity of X-rays from a GaAs layer with a thickness of $48 \mathrm{~nm}$ on Si substrate. The rms roughness of the interface of $\mathrm{GaAs}$ and $\mathrm{Si}$ was set to $0.7 \mathrm{~nm}$, the value derived from the TEM observations. The rms roughness of the GaAs surface was set to $2.8 \mathrm{~nm}$, the value derived from the AFM measurements. The agreement of the calculated and experimental results in Figure 2 is not good. The calculated result suggests the following: if the value of the surface roughness and the interfacial roughness in the calculation would be made larger, the calculated result will more closely approach the experimental result. In the TEM observation and AFM measurements, one half of the peak to peak value of the interface roughness equates to $1 \mathrm{~nm}$, and that of the GaAs surface is $4 \mathrm{~nm}$. We then recalculated the reflectivity values of this order for the surface roughness and the interface roughness in the calculation. Three calculated results for a roughness of GaAs surface of $3.5 \mathrm{~nm}, 4 \mathrm{~nm}$, and $4.5 \mathrm{~nm}$, with an interface roughness of $1 \mathrm{~nm}$ are shown in Figure 6.

Although the calculated results did more closely approach those from experiment, they still showed poor agreement. The ratio of the oscillation amplitude to the value of 


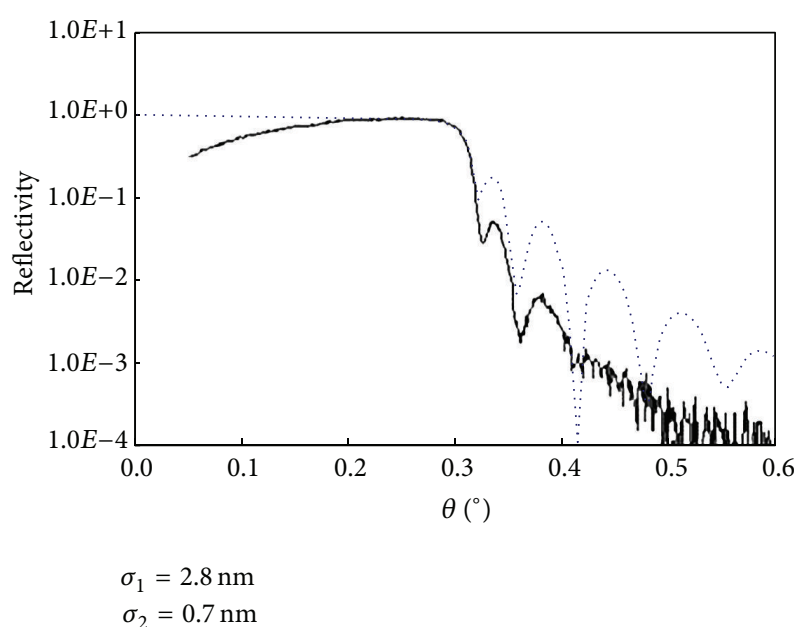

FIGURE 5: Calculated (dots) and measured (line) reflectivity from a GaAs layer with a thickness of $48 \mathrm{~nm}$ on a Si substrate. The surface roughness $\sigma_{1}$ is $2.8 \mathrm{~nm}$ and the interfacial roughness $\sigma_{2}$ is $0.7 \mathrm{~nm}$.

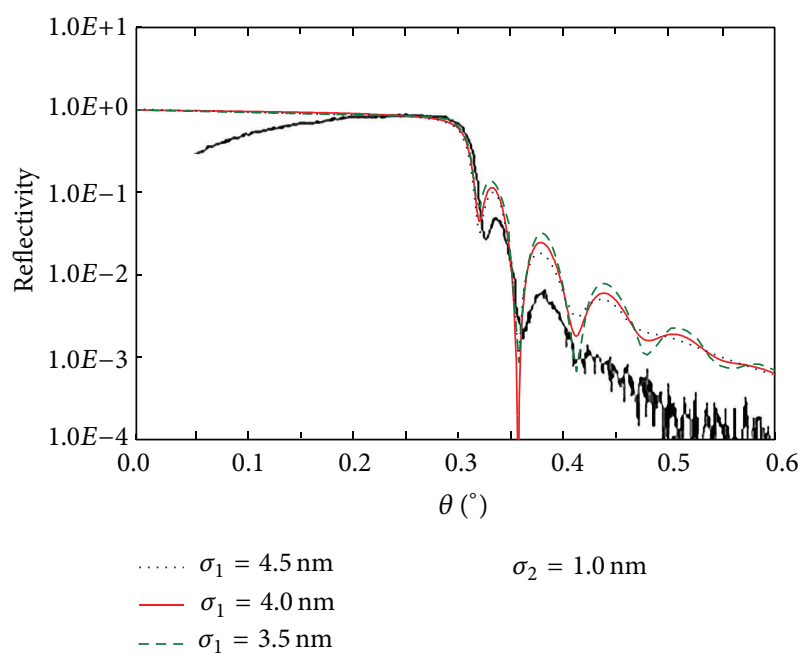

FIgURE 6: Calculated (dotted, dashed, and thin lines) and measured (thick line) reflectivity from a GaAs layer with a thickness of $48 \mathrm{~nm}$ on a $\mathrm{Si}$ substrate. In the calculation, the interface roughness $\sigma_{2}$ is $1.0 \mathrm{~nm}$. Three calculated results with the roughness $\sigma_{1}$ of GaAs surface set at $3.5 \mathrm{~nm}, 4 \mathrm{~nm}$, and $4.5 \mathrm{~nm}$ are shown.

the reflectivity near an angle of incidence of $0.36^{\circ}$ in the calculated reflectivity for the GaAs surface of $4 \mathrm{~nm}$ roughness in Figure 6 is larger than that of the reflectivity for a small roughness of $2.8 \mathrm{~nm}$ in Figure 2, that is, near an angle of incidence of $0.36^{\circ}$ interference effects appear to increase the reflectivity in the case of large roughness. It seems very strange that interference effects would operate in this way.

Figure 7 shows the reflectivity from a GaAs-covered silicon wafer, solid line shows the calculated result in the case of flat surface and flat interface, dashed line shows the calculated result in the case that the surface has an rms roughness of $4 \mathrm{~nm}$, and dotted line shows the equivalent result when the surface and interface both have an rms roughness of $4 \mathrm{~nm}$. In the latter case, the reflectivity curve (dots) decreases

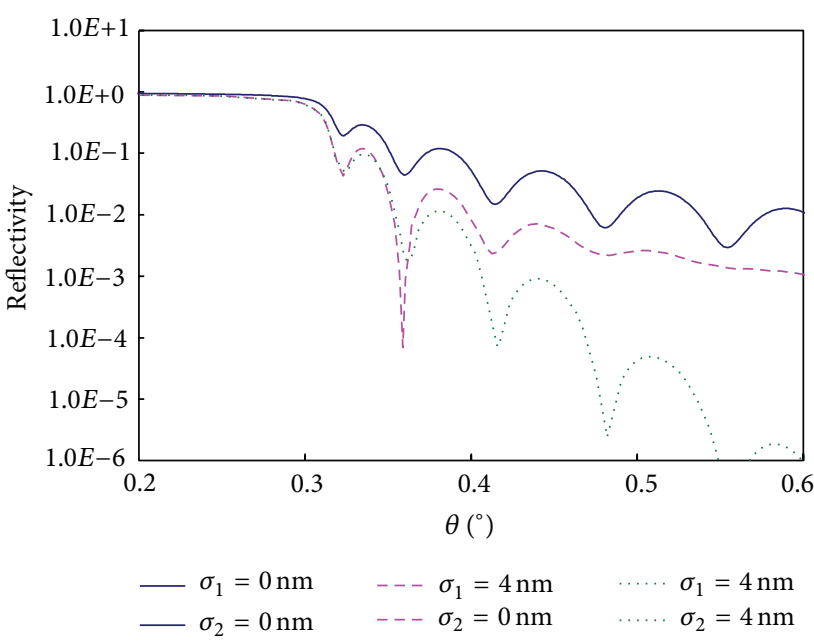

FIGURE 7: Calculated reflectivity from a GaAs layer with a thickness of $48 \mathrm{~nm}$ on a Si substrate. The solid curve is for a flat surface and a flat interface. The dashed curve is for a surface roughness $\sigma_{1}$ of $4 \mathrm{~nm}$ and a flat interface, while the dotted curve is for a surface roughness $\sigma_{1}$ of $4 \mathrm{~nm}$ and interface roughness $\sigma_{2}$ of $4 \mathrm{~nm}$.

more quickly than that in Figure 3. However, the ratio of the oscillation amplitude to the value of the reflectivity does not decrease. It seems unnatural that the effect of interference does not also decrease at a rough surface and interface, because the amount of coherent X-rays should reduce due to diffuse scattering at a rough surface and interface.

In the reflectivity curve (dashed line) for a surface roughness of $4 \mathrm{~nm}$ and with a flat interface, the ratio of the oscillation amplitude to the size of the reflectivity near an angle of incidence of $0.36^{\circ}$ is much larger than the reflectivity of the flat surface in Figure 3. It seems very strange that the interference effects would increase so much at a rough surface.

To probe these effects further, we then recalculated the reflectivity for surface roughness of $3.5 \mathrm{~nm}, 4 \mathrm{~nm}$, and $4.5 \mathrm{~nm}$, and with a flat interface. Those calculated reflectivity results are shown in Figure 8. The ratio of the oscillation amplitude to the reflectivity near an angle of incidence of $0.36^{\circ}$ in calculated reflectivity is larger in all cases than that of the reflectivity in the case of a flat surface in Figure 3.

For most angles of incidence within this range, the reflectivity of the surface with a roughness of $4 \mathrm{~nm}$ is near the mean value of the reflectivity of surfaces with roughnesses of $3.5 \mathrm{~nm}$ and $4.5 \mathrm{~nm}$. However, near an angle of incidence of $0.36^{\circ}$, the reflectivity of the surface with a roughness of $4 \mathrm{~nm}$ is very much attenuated compared to that same average. It seems very strange that the reflectivity of the average roughness has a value quite different from the mean value of the reflectivity of each roughness, because the value of the roughness is not the value of the amplitude of a rough surface but the standard deviation value of various amplitudes of rough surface.

Figure 9 shows the reflectivity from a tungsten-covered silicon wafer calculated by the theory in use prior to this work. The ratio of the oscillation amplitude to the value of the reflectivity from a surface with an rms surface roughness of $0.3 \mathrm{~nm}$ (dashed line) does not decrease near an angle of 


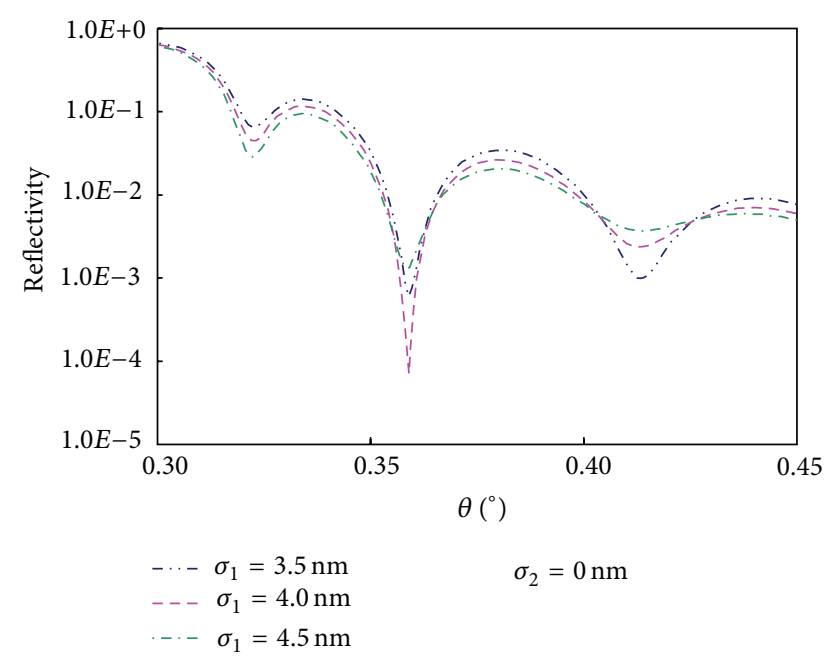

FIGURE 8: Calculated reflectivity from a GaAs layer with a thickness of $48 \mathrm{~nm}$ on a Si substrate. In the calculation, the interface roughness $\sigma_{2}$ is $0 \mathrm{~nm}$. Three calculated results are shown for a GaAs surface with an rms roughness $\sigma_{1}$ of $3.5 \mathrm{~nm}, 4 \mathrm{~nm}$, and $4.5 \mathrm{~nm}$.

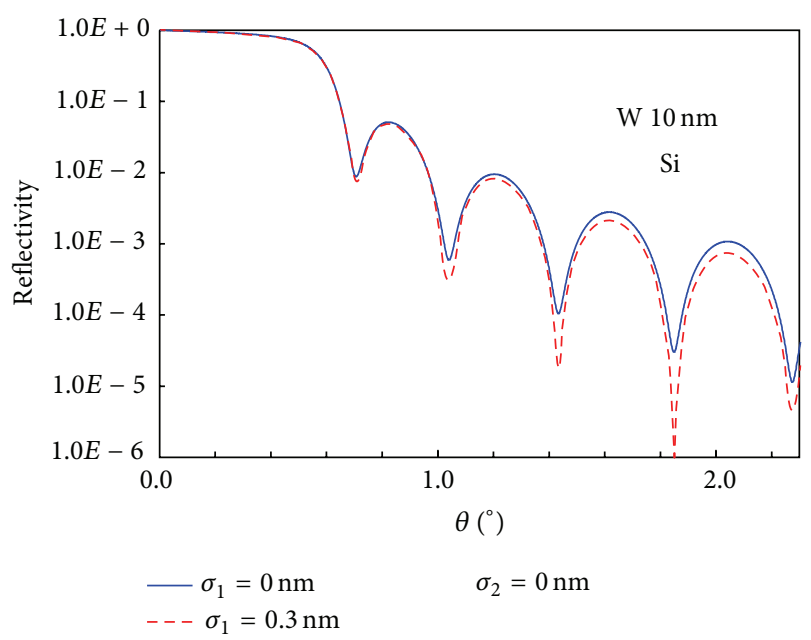

FIGURE 9: X-ray reflectivity from a silicon wafer covered with a thin $(10 \mathrm{~nm})$ tungsten film calculated by the theory in use prior to this work. Solid line shows the case of a flat surface. Dashed line shows the case of a surface with an rms surface roughness of $0.3 \mathrm{~nm}$.

incidence of $1.8^{\circ}$ but increases. This result is strange and not reasonable.

3.3. Effect of Roughness on X-Ray Reflectivity of Multilayer Surface. We now consider the previous strange result of the $\mathrm{X}$-ray reflectivity which was calculated based on the Parratt formalism [1] with the use of the Nevot and Croce approach to account for roughness [2]. In that calculation, the $\mathrm{X}$ ray reflectivity is derived using the relation of the reflection coefficient $R_{j-1, j}$ and $R_{j, j+1}$ as follows:

$$
R_{j-1, j}=\frac{R_{j, j+1}+\Psi_{j-1, j}^{\prime}}{1+R_{j, j+1} \Psi_{j-1, j}^{\prime}} \exp \left(2 i k_{j-1, z} h_{j-1}\right),
$$

where the reduced Fresnel reflection coefficient $\Psi^{\prime}$ that takes into account the effect of the roughness is as follows:

$$
\Psi_{j, j-1}^{\prime}=\Psi_{j, j-1} \exp \left(-2 k_{j, z} k_{j-1, z} \sigma_{j, j-1}^{2}\right) .
$$

However, the relationship between the reflection coefficients $R_{j-1, j}$ and $R_{j, j+1}$ was originally derived as the following equation:

$$
\begin{aligned}
\mathbf{R}_{j-1, j}= & \frac{\Psi_{j-1, j}^{\prime}+\left(\boldsymbol{\Phi}_{j-1, j}^{\prime} \boldsymbol{\Phi}_{j, j-1}^{\prime}-\Psi_{j-1, j}^{\prime} \Psi_{j, j-1}^{\prime}\right) \mathbf{R}_{j, j+1}}{1-\Psi_{j, j-1}^{\prime} \mathbf{R}_{j, j+1}} \\
& \times \exp \left(2 i k_{j-1, z} h_{j-1}\right) .
\end{aligned}
$$

Here, the following conditional relations between the Fresnel coefficient for reflection and refraction are relevant to the previous equation:

$$
\begin{gathered}
\boldsymbol{\Phi}_{j-1, j}^{\prime} \boldsymbol{\Phi}_{j, j-1}^{\prime}-\boldsymbol{\Psi}_{j-1, j}^{\prime} \boldsymbol{\Psi}_{j, j-1}^{\prime}=1, \\
\boldsymbol{\Psi}_{j-1, j}^{\prime}=-\boldsymbol{\Psi}_{j, j-1}^{\prime},
\end{gathered}
$$

then,

$$
\Phi_{j-1, j}^{\prime} \Phi_{j, j-1}^{\prime}+\Psi_{j, j-1}^{\prime 2}=1
$$

that is,

$$
\boldsymbol{\Phi}_{j-1, j}^{\prime} \Phi_{j, j-1}^{\prime}=1-\Psi_{j, j-1}^{\prime 2} .
$$

The Fresnel coefficients for refraction at the rough interface are derived using the Fresnel reflection coefficient $\Psi$ as follows:

$$
\begin{aligned}
\boldsymbol{\Phi}_{j-1, j}^{\prime} \boldsymbol{\Phi}_{j, j-1}^{\prime}-\boldsymbol{\Phi}_{j-1, j} \boldsymbol{\Phi}_{j, j-1} \\
=\boldsymbol{\Psi}_{j, j-1}^{2}\left(1-\exp \left(-2 k_{j, z} k_{j-1, z} \sigma_{j, j-1}^{2}\right)\right)>0, \\
\boldsymbol{\Phi}_{j-1, j}^{\prime} \boldsymbol{\Phi}_{j, j-1}^{\prime} \\
=\boldsymbol{\Phi}_{j-1, j} \boldsymbol{\Phi}_{j, j-1}+\left(1-\boldsymbol{\Phi}_{j-1, j} \boldsymbol{\Phi}_{j, j-1}\right) \\
\quad \times\left(1-\exp \left(-2 k_{j, z} k_{j-1, z} \sigma_{j, j-1}^{2}\right)\right) .
\end{aligned}
$$

Therefore, the Fresnel coefficients for refraction at the rough interface are necessarily larger than the Fresnel coefficient for refraction at the flat interface. The resulting increase in the transmission coefficient completely overpowers any decrease in the value of the reflection coefficient. These coefficients for refraction obviously contain a mistake because the penetration of X-rays should decrease at a rough interface because of diffuse scattering. We propose that the unnatural results in the previous calculation of the X-ray reflectivity originate from the fact that diffuse scattering was not considered. In fact (52) contains the X-ray energy conservation rule at the interface as the following identity equation for the Fresnel coefficient:

$$
\Phi_{j-1, j} \Phi_{j, j-1}-\Psi_{j-1, j} \Psi_{j, j-1}=\Phi_{j-1, j} \Phi_{j, j-1}+\Psi_{j-1, j}^{2}=1 .
$$


Here, we consider the energy flow of the X-ray. In electromagnetic radiation, $\mathbf{E}, \mathbf{H}$, the energy flow is equal to the Poynting vector

$$
\mathbf{p}=\frac{1}{4}\left(\mathbf{E}^{*} \times \mathbf{H}+\mathbf{E} \times \mathbf{H}^{*}\right),
$$

where

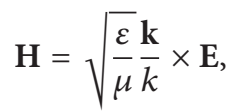

and $\varepsilon$ and $\mu$ are the dielectric and magnetic permeability. The Poynting vector is therefore

$$
\begin{aligned}
\mathbf{p} & =\frac{1}{4} \sqrt{\frac{\bar{\varepsilon}}{\mu}}\left(\mathbf{E}^{*} \times\left(\frac{\mathbf{k}}{k} \times \mathbf{E}\right)+\mathbf{E} \times\left(\frac{\mathbf{k}}{k} \times \mathbf{E}\right)^{*}\right) \\
& =\frac{1}{4} \sqrt{\frac{\varepsilon}{\mu}}\left(\frac{\mathbf{k}}{k} \mathbf{E}^{*} \cdot \mathbf{E}+\frac{\mathbf{k}^{*}}{k} \mathbf{E} \cdot \mathbf{E}^{*}\right)=\frac{1}{2 \omega \mu} \frac{\mathbf{k}+\mathbf{k}^{*}}{2}|\mathbf{E}|^{2} .
\end{aligned}
$$

Then, the Poynting vector that crosses the interface is

$$
\begin{aligned}
\int \mathbf{p} d \mathbf{S} & =\int \frac{1}{2 \mu \omega} \frac{\mathbf{k}+\mathbf{k}^{*}}{2}|\mathbf{E}|^{2} d \mathbf{S} \\
& =\frac{1}{2 \mu \omega} \int \frac{\mathbf{k}+\mathbf{k}^{*}}{2}|\mathbf{E}|^{2} d \mathbf{S}=\frac{1}{2 \mu \omega} \frac{k_{z}+k_{z}^{*}}{2}|\mathbf{A}|^{2} .
\end{aligned}
$$

The amplitudes $\mathbf{A}_{j-1}$ and $\mathbf{A}_{j-1}^{\prime}$ of the electric fields $\mathbf{E}_{j-1}$, $\mathbf{E}_{j-1}^{\prime}$ at the $j$ th layer and amplitudes $\mathbf{A}_{j}$ and $\mathbf{A}_{j}^{\prime}$ of the electric fields $\mathbf{E}_{j}, \mathbf{E}_{j}^{\prime}$ at the $(j+1)$ th layer are related by the following equations:

$$
\begin{aligned}
& \left(\begin{array}{cc}
\boldsymbol{\Phi}_{j-1, j} & 0 \\
0 & \boldsymbol{\Phi}_{j-1, j}
\end{array}\right)\left(\begin{array}{c}
\mathbf{A}_{j-1} \\
\mathbf{A}_{j-1}^{\prime}
\end{array}\right) \\
& =\left(\begin{array}{cc}
\exp \left(-i k_{j-1, z} h_{j-1}\right) & 0 \\
0 & \exp \left(i k_{j-1, z} h_{j-1}\right)
\end{array}\right) \\
& \quad \times\left(\begin{array}{cc}
1 & -\Psi_{j, j-1} \\
\boldsymbol{\Psi}_{j-1, j} & \left(\boldsymbol{\Phi}_{j-1, j} \boldsymbol{\Phi}_{j, j-1}-\boldsymbol{\Psi}_{j-1, j} \boldsymbol{\Psi}_{j, j-1}\right.
\end{array}\right)\left(\begin{array}{l}
\mathbf{A}_{j} \\
\mathbf{A}_{j}^{\prime}
\end{array}\right) .
\end{aligned}
$$

When

$$
\begin{gathered}
\boldsymbol{\Phi}_{j-1, j} \boldsymbol{\Phi}_{j, j-1}-\boldsymbol{\Psi}_{j-1, j} \boldsymbol{\Psi}_{j, j-1}=1, \\
\boldsymbol{\Psi}_{j-1, j}=-\boldsymbol{\Psi}_{j, j-1},
\end{gathered}
$$

we can describe the previous equation as

$$
\begin{aligned}
& \left(\begin{array}{cc}
\boldsymbol{\Phi}_{j-1, j} & 0 \\
0 & \boldsymbol{\Phi}_{j-1, j}
\end{array}\right)\left(\begin{array}{cc}
\mathbf{A}_{j-1} & \mathbf{A}_{j-1}^{\prime *} \\
\mathbf{A}_{j-1}^{\prime} & \mathbf{A}_{j-1}^{*}
\end{array}\right) \\
& =\left(\begin{array}{cc}
\exp \left(-i k_{j-1, z} h_{j-1}\right) & 0 \\
0 & \exp \left(i k_{j-1, z} h_{j-1}\right)
\end{array}\right) \\
& \times\left(\begin{array}{cc}
1 & \boldsymbol{\Psi}_{j-1, j} \\
\Psi_{j-1, j} & 1
\end{array}\right)\left(\begin{array}{cc}
\mathbf{A}_{j} & \mathbf{A}_{j}^{\prime *} \\
\mathbf{A}_{j}^{\prime} & \mathbf{A}_{j}^{*}
\end{array}\right) .
\end{aligned}
$$

From the determinant of the refraction matrix,

$$
\begin{aligned}
& \left|\begin{array}{cc}
\boldsymbol{\Phi}_{j-1, j} & 0 \\
0 & \boldsymbol{\Phi}_{j-1, j}
\end{array}\right|\left(\left|\mathbf{A}_{j-1}\right|^{2}-\left|\mathbf{A}_{j-1}^{\prime}\right|^{2}\right) \\
& \quad=\left|\begin{array}{cc}
\exp \left(-i k_{j-1, z} h_{j-1}\right) & 0 \\
0 & \exp \left(i k_{j-1, z} h_{j-1}\right)
\end{array}\right| \\
& \quad \times\left|\begin{array}{cc}
1 & \boldsymbol{\Psi}_{j-1, j} \\
\boldsymbol{\Psi}_{j-1, j} & 1
\end{array}\right|\left(\left|\mathbf{A}_{j}\right|^{2}-\left|\mathbf{A}_{j}^{\prime}\right|^{2}\right) .
\end{aligned}
$$

Then

$$
\begin{gathered}
\boldsymbol{\Phi}_{j-1, j}^{2}\left(\left|\mathbf{A}_{j-1}\right|^{2}-\left|\mathbf{A}_{j-1}^{\prime}\right|^{2}\right) \\
=\left(1-\Psi_{j-1, j}^{2}\right)\left(\left|\mathbf{A}_{j}\right|^{2}-\left|\mathbf{A}_{j}^{\prime}\right|^{2}\right), \\
\boldsymbol{\Phi}_{j-1, j}\left(\left|\mathbf{A}_{j-1}\right|^{2}-\left|\mathbf{A}_{j-1}^{\prime}\right|^{2}\right)=\boldsymbol{\Phi}_{j, j-1}\left(\left|\mathbf{A}_{j}\right|^{2}-\left|\mathbf{A}_{j}^{\prime}\right|^{2}\right), \\
\frac{2 k_{j-1, z}}{k_{j-1, z}+k_{j, z}}\left(\left|\mathbf{A}_{j-1}\right|^{2}-\left|\mathbf{A}_{j-1}^{\prime}\right|^{2}\right) \\
=\frac{2 k_{j, z}}{k_{j-1, z}+k_{j, z}}\left(\left|\mathbf{A}_{j}\right|^{2}-\left|\mathbf{A}_{j}^{\prime}\right|^{2}\right), \\
k_{j-1, z}\left|\mathbf{A}_{j-1}\right|^{2}-k_{j-1, z}\left|\mathbf{A}_{j-1}^{\prime}\right|^{2}=k_{j, z}\left|\mathbf{A}_{j}\right|^{2}-k_{j, z}\left|\mathbf{A}_{j}^{\prime}\right|^{2} .
\end{gathered}
$$

That is, the X-ray energy flow is conserved at the interface. When the Fresnel coefficients at the rough interface obeys the following equations,

$$
\begin{gathered}
\boldsymbol{\Phi}_{j-1, j}^{\prime} \boldsymbol{\Phi}_{j, j-1}^{\prime}-\boldsymbol{\Psi}_{j-1, j}^{\prime} \boldsymbol{\Psi}_{j, j-1}^{\prime}=1, \\
\boldsymbol{\Psi}_{j-1, j}^{\prime}=-\boldsymbol{\Psi}_{j, j-1}^{\prime},
\end{gathered}
$$

these coefficients fulfil X-ray energy flow conservation at the interface, and so diffuse scattering was not considered at the rough interface.

This conservation expression should not apply any longer when the Fresnel reflection coefficient is replaced by the reduced coefficient $\Psi^{\prime}$ when there is roughening at the interface. Therefore, calculating the reflectivity using this reduced Fresnel reflection coefficient $\Psi^{\prime}$ in (50) will incorrectly increase the Fresnel transmission coefficient $\Phi^{\prime}$; that is, $\Phi<\Phi^{\prime}$.

The penetration of X-rays should decrease at a rough interface because of diffuse scattering. Therefore, the identity equation for the Fresnel coefficients become

$$
\begin{gathered}
\Phi_{j-1, j}^{\prime} \Phi_{j, j-1}^{\prime}-\Psi_{j-1, j}^{\prime} \Psi_{j, j-1}^{\prime} \\
=\Phi_{j-1, j}^{\prime} \Phi_{j, j-1}^{\prime}+\Psi_{j-1, j}^{\prime 2} \\
=1-D^{2}<1,
\end{gathered}
$$

where $D^{2}$ is a decrease due to diffuse scattering. Then, in the calculation of X-ray reflectivity when there is roughening at the surface or the interface, the Fresnel transmission 
coefficient $\Phi^{\prime}$ should be used for the reduced coefficient. Several theories exist to describe the influence of roughness on X-ray scattering [8-19]. When the surface and interface are both rough, the Fresnel coefficient for refraction has been derived in several theories [14-19].

\subsection{The Refractive Fresnel Coefficient of a Rough Interface Used} in Previous Reflectivity Calculations. Initially, we consider the reduced Fresnel coefficient, which is known as the CroceNevot factor. When the $z$-position of the interface of 0 th layer and 1th layer $z_{0,1}$ fluctuates vertically as a function of the lateral position because of the interface roughness, the relations between theamplitudes $A_{0}, A_{0}^{\prime}, A_{1}$, and $A_{1}^{\prime}$ are derived by the use of the Fresnel coefficient tensor $\boldsymbol{\Phi}$ for refraction and the Fresnel coefficient tensor $\Psi$ for reflection as follows:

$$
\begin{aligned}
\mathbf{A}_{1} & \exp \left(i k_{1, z} z_{0,1}\right) \\
& =\boldsymbol{\Phi}_{0,1} \mathbf{A}_{0} \exp \left(i k_{0, z} z_{0,1}\right)+\boldsymbol{\Psi}_{1,0} \mathbf{A}_{1}^{\prime} \exp \left(-i k_{1, z} z_{0,1}\right), \\
\mathbf{A}_{0}^{\prime} & \exp \left(-i k_{0, z} z_{0,1}\right) \\
& =\boldsymbol{\Psi}_{0,1} \mathbf{A}_{0} \exp \left(i k_{0, z} z_{0,1}\right)+\boldsymbol{\Phi}_{1,0} \mathbf{A}_{1}^{\prime} \exp \left(-i k_{1, z} z_{0,1}\right) .
\end{aligned}
$$

Then,

$$
\begin{aligned}
& \left(\begin{array}{cc}
\boldsymbol{\Phi}_{0,1} \exp \left(i k_{0, z} z_{0,1}\right) & 0 \\
\boldsymbol{\Psi}_{0,1} \exp \left(i k_{0, z} z_{0,1}\right) & -\exp \left(-i k_{0, z} z_{0,1}\right)
\end{array}\right)\left(\begin{array}{c}
A_{0} \\
A_{0}^{\prime}
\end{array}\right)=\left(\begin{array}{cc}
\exp \left(i k_{1, z} z_{0,1}\right) & -\boldsymbol{\Psi}_{1,0} \exp \left(-i k_{1, z} z_{0,1}\right) \\
0 & -\boldsymbol{\Phi}_{1,0} \exp \left(-i k_{1, z} z_{0,1}\right)
\end{array}\right)\left(\begin{array}{c}
A_{1} \\
A_{1}^{\prime}
\end{array}\right) \\
& \left(\begin{array}{c}
A_{0} \\
A_{0}^{\prime}
\end{array}\right)=\frac{1}{\boldsymbol{\Phi}_{0,1}}\left(\begin{array}{cc}
\exp \left(-i k_{0, z} z_{0,1}\right) & 0 \\
\boldsymbol{\Psi}_{0,1} \exp \left(i k_{0, z} z_{0,1}\right) & -\boldsymbol{\Phi}_{0,1} \exp \left(i k_{0, z} z_{0,1}\right)
\end{array}\right)\left(\begin{array}{cc}
\exp \left(i k_{1, z} z_{0,1}\right) & -\boldsymbol{\Psi}_{1,0} \exp \left(-i k_{1, z} z_{0,1}\right) \\
0 & -\boldsymbol{\Phi}_{1,0} \exp \left(-i k_{1, z} z_{0,1}\right)
\end{array}\right)\left(\begin{array}{c}
A_{1} \\
A_{1}^{\prime}
\end{array}\right) \\
& \left(\begin{array}{c}
A_{0} \\
A_{0}^{\prime}
\end{array}\right)=\frac{1}{\boldsymbol{\Phi}_{0,1}}\left(\begin{array}{cc}
\exp \left(-i\left(k_{0, z}-k_{1, z}\right) z_{0,1}\right) & -\boldsymbol{\Psi}_{1,0} \exp \left(-i\left(k_{0, z}+k_{1, z}\right) z_{0,1}\right) \\
\boldsymbol{\Psi}_{0,1} \exp \left(i\left(k_{0, z}+k_{1, z}\right) z_{0,1}\right) & \left(\boldsymbol{\Phi}_{0,1} \boldsymbol{\Phi}_{1,0}-\boldsymbol{\Psi}_{0,1} \boldsymbol{\Psi}_{1,0}\right) \exp \left(i\left(k_{0, z}-k_{1, z}\right) z_{0,1}\right)
\end{array}\right)\left(\begin{array}{c}
A_{1} \\
A_{1}^{\prime}
\end{array}\right),
\end{aligned}
$$

where

$$
\begin{array}{ll}
\Psi_{0,1, y y}=\frac{k_{0, z}-k_{1, z}}{k_{0, z}+k_{1, z}}, & \Psi_{1,0 y y}=\frac{k_{1, z}-k_{0, z}}{k_{0, z}+k_{1, z}}, \\
\Phi_{0,1, y y}=\frac{2 k_{0, z}}{k_{0, z}+k_{1, z}}, & \Phi_{1,0, y y}=\frac{2 k_{1, z}}{k_{1, z}+k_{0, z}} .
\end{array}
$$

Then

$$
\left(\begin{array}{c}
A_{0} \\
A_{0}^{\prime}
\end{array}\right)=\left(\begin{array}{cc}
\frac{k_{0, z}+k_{1, z}}{2 k_{0, z}} \exp \left(-i\left(k_{0, z}-k_{1, z}\right) z_{0,1}\right) & \frac{k_{0, z}-k_{1, z}}{2 k_{0, z}} \exp \left(-i\left(k_{0, z}+k_{1, z}\right) z_{0,1}\right) \\
\frac{k_{0, z}-k_{1, z}}{2 k_{0, z}} \exp \left(i\left(k_{0, z}+k_{1, z}\right) z_{0,1}\right) & \frac{k_{0, z}+k_{1, z}}{2 k_{0, z}} \exp \left(i\left(k_{0, z}-k_{1, z}\right) z_{0,1}\right)
\end{array}\right)\left(\begin{array}{c}
A_{1} \\
A_{1}^{\prime}
\end{array}\right) .
$$

We take the average value of the matrix over the whole area coherently illuminated by the incident X-ray beam. This leads to

$$
\left(\begin{array}{c}
A_{0} \\
A_{0}^{\prime}
\end{array}\right)=\left(\begin{array}{ll}
\frac{k_{0, z}+k_{1, z}}{2 k_{0, z}}\left\langle\exp \left(-i\left(k_{0, z}-k_{1, z}\right) z_{0,1}\right)\right\rangle & \frac{k_{0, z}-k_{1, z}}{2 k_{0, z}}\left\langle\exp \left(-i\left(k_{0, z}+k_{1, z}\right) z_{0,1}\right)\right\rangle \\
\frac{k_{0, z}-k_{1, z}}{2 k_{0, z}}\left\langle\exp \left(i\left(k_{0, z}+k_{1, z}\right) z_{0,1}\right)\right\rangle & \frac{k_{0, z}+k_{1, z}}{2 k_{0, z}}\left\langle\exp \left(i\left(k_{0, z}-k_{1, z}\right) z_{0,1}\right)\right\rangle
\end{array}\right)\left(\begin{array}{c}
A_{1} \\
A_{1}^{\prime}
\end{array}\right) .
$$


For Gaussian statistics of standard deviation value $\sigma$,

$$
\begin{gathered}
g(z)=\frac{1}{\sqrt{2 \pi} \sigma} \exp \left(-\frac{z^{2}}{2 \sigma^{2}}\right), \\
\langle f(z)\rangle=\int_{-\infty}^{\infty} g(z) f(z) d z=\int_{-\infty}^{\infty} \frac{1}{\sqrt{2 \pi} \sigma} \exp \left(-\frac{z^{2}}{2 \sigma^{2}}\right) f(z) d z, \\
\left\langle\exp \left(i k z_{0,1}\right)\right\rangle=\int_{-\infty}^{\infty} g\left(z_{0,1}\right) \exp \left(i k z_{0,1}\right) d z_{0,1} \\
=\int_{-\infty}^{\infty} \frac{1}{\sqrt{2 \pi} \sigma_{0,1}} \exp \left(-\frac{z_{0,1}^{2}}{2 \sigma_{0,1}^{2}}\right) \exp \left(i k z_{0,1}\right) d z_{0,1} \\
=\exp \left(-\frac{1}{2} k^{2} \sigma_{0,1}^{2}\right), \\
\left(\begin{array}{c}
A_{0} \\
A_{0}^{\prime}
\end{array}\right)=\left(\begin{array}{cc}
\frac{k_{0, z}+k_{1, z}}{2 k_{0, z}} \exp \left(-\frac{1}{2}\left(k_{0, z}-k_{1, z}\right)^{2} \sigma_{0,1}^{2}\right) & \frac{k_{0, z}-k_{1, z}}{2 k_{0, z}} \exp \left(-\frac{1}{2}\left(k_{0, z}+k_{1, z}\right)^{2} \sigma_{0,1}^{2}\right) \\
\frac{k_{0, z}-k_{1, z}}{2 k_{0, z}} \exp \left(-\frac{1}{2}\left(k_{0, z}+k_{1, z}\right)^{2} \sigma_{0,1}^{2}\right) & \frac{k_{0, z}+k_{1, z}}{2 k_{0, z}} \exp \left(-\frac{1}{2}\left(k_{0, z}-k_{1, z}\right)^{2} \sigma_{0,1}^{2}\right)
\end{array}\right)\left(\begin{array}{c}
A_{1} \\
A_{1}^{\prime}
\end{array}\right) .
\end{gathered}
$$

Therefore

$$
\begin{aligned}
& \left(\begin{array}{l}
A_{0}^{\prime} \\
A_{1}
\end{array}\right) \\
& =\left(\begin{array}{cc}
\frac{k_{0, z}-k_{1, z}}{k_{0, z}+k_{1, z}} \exp \left(-2 k_{0, z} k_{1, z} \sigma_{0,1}^{2}\right) & \boldsymbol{\Phi}_{1,0}^{\prime} \\
\frac{2 k_{0, z}}{k_{0, z}+k_{1, z}} \exp \left(\frac{1}{2}\left(k_{0, z}-k_{1, z}\right)^{2} \sigma_{0,1}^{2}\right) & \frac{k_{1, z}-k_{0, z}}{k_{0, z}+k_{1, z}} \exp \left(-2 k_{0, z} k_{1, z} \sigma_{0,1}^{2}\right)
\end{array}\right) \\
& \times\left(\begin{array}{c}
A_{0} \\
A_{1}^{\prime}
\end{array}\right)
\end{aligned}
$$

where $\Phi_{1,0}^{\prime}=2 k_{1, z} /\left(k_{0, z}+k_{1, z}\right) \exp \left(1 / 2\left(k_{0, z}-k_{1, z}\right)^{2} \sigma_{0,1}^{2}\right)$ $\left(\left(k_{0, z}+k_{1, z}\right)^{2} /\left(4 k_{0, z} k_{1, z}\right) \exp \left(-\left(k_{0, z}-k_{1, z}\right)^{2} \sigma_{0,1}^{2}\right)-\left(k_{0, z}-\right.\right.$ $\left.\left.k_{1, z}\right)^{2} /\left(4 k_{0, z} k_{1, z}\right) \exp \left(-\left(k_{0, z}+k_{1, z}\right)^{2} \sigma_{0,1}^{2}\right)\right)$. Then the Fresnel reflection coefficients $\Psi^{\prime}$ are reduced as follows:

$$
\begin{gathered}
\Psi_{0,1}^{\prime}=\Psi_{0,1} \exp \left(-2 k_{0, z} k_{1, z} \sigma_{0,1}^{2}\right), \\
\Psi_{1,0}^{\prime}=-\Psi_{0,1} .
\end{gathered}
$$

However, the Fresnel refraction coefficients $\Phi^{\prime}$ increase as follows:

$$
\boldsymbol{\Phi}_{0,1}^{\prime}=\boldsymbol{\Phi}_{0,1} \exp \left(\frac{1}{2}\left(k_{0, z}-k_{1, z}\right)^{2} \sigma_{0,1}^{2}\right)
$$

$$
\begin{aligned}
& \boldsymbol{\Phi}_{1,0}^{\prime}= \boldsymbol{\Phi}_{1,0} \exp \left(\frac{1}{2}\left(k_{0, z}-k_{1, z}\right)^{2} \sigma_{0,1}^{2}\right) \\
& \times\left(\frac{\left(k_{0, z}+k_{1, z}\right)^{2}}{4 k_{0, z} k_{1, z}} \exp \left(-\left(k_{0, z}-k_{1, z}\right)^{2} \sigma_{0,1}^{2}\right)\right. \\
&\left.\quad-\frac{\left(k_{0, z}-k_{1, z}\right)^{2}}{4 k_{0, z} k_{1, z}} \exp \left(-\left(k_{0, z}+k_{1, z}\right)^{2} \sigma_{0,1}^{2}\right)\right) .
\end{aligned}
$$

The modified Fresnel refraction coefficients $\Phi_{0,1}^{\prime}$ corresponds to equation (10.29) in p.200 of Holy et al. [14], 
equation (8.24) in p.242 of Daillant and Gibaud [15], and equation (1.117) in p.29 of Sakurai [20]. However, no one obtained the expression corresponding to $\Phi_{1,0}^{\prime}$. It is peculiar that $\Phi_{1,0}^{\prime}$ and $\Phi_{0,1}^{\prime}$ are asymmetrical. It comes to cause a different result if 1 th layer and 0th layers are replaced and calculated. Therefore this derived $\Phi^{\prime}$ should not be used to calculate the reflectivity from rough surfaces and interfaces.

The derived Fresnel refraction coefficients $\Phi^{\prime}$ increase. This increase in the transmission coefficient completely overpowers any decrease in the value of the reflection coefficient as the following:

$$
\begin{gathered}
\boldsymbol{\Phi}_{0,1}^{\prime} \boldsymbol{\Phi}_{1,0}^{\prime}-\boldsymbol{\Psi}_{0,1}^{\prime} \boldsymbol{\Psi}_{1,0}^{\prime}=1, \\
\boldsymbol{\Phi}_{j-1, j}^{\prime} \boldsymbol{\Phi}_{j, j-1}^{\prime}-\boldsymbol{\Phi}_{j-1, j} \boldsymbol{\Phi}_{j, j-1} \\
=\boldsymbol{\Psi}_{j, j-1}^{2}\left(1-\exp \left(-2 k_{j, z} k_{j-1, z} \sigma_{j, j-1}^{2}\right)\right)>0 .
\end{gathered}
$$

Moreover, if the deformation modulus of $\Phi_{1,0}^{\prime}$ is assumed to be $\Phi_{0,1}^{\prime}$, the left side of $(80)$ exceeds unity and therefore (78) is obviously wrong.

In Nevot and Croce's treatment of the Parratt formalism for the reflectivity calculation including surface and interface roughness [2], the relations of the Fresnel coefficients between reflection and transmission as (52), (68), and (80) were not shown. Furthermore, the modification of the Fresnel coefficients according to Nevot and Croce has been used for only surface and interface reflection. However, the modification of the transmission coefficients has an important role when the roughness of the surface or interface is high, and the effect of diffuse scattering due to that roughness should not be ignored, as shown in (69). The error in Nevot and Croce's treatment [2] originates in the fact that the modified Fresnel coefficients was calculated based on the Parratt formalism which contains the X-ray energy conservation rule at the surface and interface. In the discussion on pp.767768 of Nevot and Croce's [2], their Fresnel coefficients at the rough interface fulfil X-ray energy flow conservation at the interface, and so diffuse scattering was ignored at the rough interface. In their discussion, the transmission coefficients $t_{R}$ and $t_{I}$ were replaced approximately by the reflection coefficients $r_{R}$ and $r_{I}$ by the ignoring diffuse scattering term, and according to the principle of conservation energy. The reflection coefficient $r_{R}$ at the rough interface should be expressed as a function of the reflection coefficient $r_{I}$ and transmission coefficient $t_{I}$. However, the reflection coefficient $r_{R}$ at the rough interface was expressed only by the reflection coefficient $r_{I}$, while the transmission coefficient $t_{I}$ had already been replaced by the reflection coefficient $r_{I}$ by the ignoring diffuse scattering term in the relationship based on the principle of the conservation of energy. Thus, the reflection coefficient $r_{R}$ at the rough interface as equation (11) of p.771 in Nevot and Croce [2] had been expressed with the reflection coefficient $r_{I}$ only, and this results in the equation was also sure to include the conservation of energy.

The resulting increase in the transmission coefficient completely overpowers any decrease in the value of the reflection coefficient at the rough interface. Thus, because Nevot and Croce's treatment of the Parratt formalism contains a fundamental mistake regardless of the size of the roughness, results using this approach cannot be correct. The size of the modification of the transmission coefficient is one-order smaller than that of reflection coefficient, but the size of transmission coefficient is one-order larger than the reflection coefficient at angles larger than critical angle. Thus, the errors of transmittance without the modification cannot be ignored.

Of course, there are cases where that Nevot and Croce's treatment can be applied. However, their method can be applied only to the case where there is no density distribution change at all in the direction parallel to the surface on the surface field side, and only when the scattering vector is normal to the surface. A typical example of surface medium to which this model can be applied is one where only the density distribution change in the vertical direction to the surface exists, as caused by diffusion, and so forth. In such a special case, Nevot and Croce's treatment can be applied without any problem. However, because a general multilayer film always has structure in a direction parallel to the surface field side, Nevot and Croce's expression fails even when the roughness is extremely small. The use of only Fresnel reflection coefficients by Nevot and Croce is a fundamental mistake that does not depend on the size of the roughness.

3.5. The Refractive Fresnel Coefficient of a Rough Interface Used in New Reflectivity Calculations. To proceed, we therefore reconsider the derivation of the average value of the matrix as the same derivation of (70) when we consider the reduced Fresnel coefficient, which is known as the Croce-Nevot factor.

When the $z$-position of the interface of the 0th layer and 1th layer $z_{0,1}$ fluctuates vertically as a function of the lateral position because of the interface roughness, the relations between the electric fields are derived by the use of the Fresnel coefficient tensor $\Phi$ for refraction and the Fresnel coefficient tensor $\Psi$ for reflection as follows:

$$
\begin{aligned}
& \mathbf{E}_{1}\left(z_{0,1}\right)=\boldsymbol{\Phi}_{0,1} \mathbf{E}_{0}\left(z_{0,1}\right)+\boldsymbol{\Psi}_{1,0} \mathbf{E}_{1}^{\prime}\left(z_{0,1}\right), \\
& \mathbf{E}_{0}^{\prime}\left(z_{0,1}\right)=\boldsymbol{\Psi}_{0,1} \mathbf{E}_{0}\left(z_{0,1}\right)+\boldsymbol{\Phi}_{1,0} \mathbf{E}_{1}^{\prime}\left(z_{0,1}\right),
\end{aligned}
$$

where

$$
\begin{aligned}
& \mathbf{E}_{0}\left(z_{0,1}\right)=\mathbf{E}_{0}(0) \exp \left[i k_{0, z} z_{0,1}\right], \\
& \mathbf{E}_{0}^{\prime}(0)=\mathbf{E}_{0}^{\prime}\left(z_{0,1}\right) \exp \left[i k_{0, z} z_{0,1}\right], \\
& \mathbf{E}_{1}\left(z_{0,1}\right)=\mathbf{E}_{1}(0) \exp \left[i k_{1, z} z_{0,1}\right], \\
& \mathbf{E}_{1}^{\prime}(0)=\mathbf{E}_{1}^{\prime}\left(z_{0,1}\right) \exp \left[i k_{1, z} z_{0,1}\right] .
\end{aligned}
$$


Then

$$
\begin{aligned}
& \mathbf{E}_{1}(0) \exp \left[i k_{1, z} z_{0,1}\right] \\
&= \boldsymbol{\Phi}_{0,1} \mathbf{E}_{0}(0) \exp \left[i k_{0, z} z_{0,1}\right] \\
&+\boldsymbol{\Psi}_{1,0} \mathbf{E}_{1}^{\prime}(0) \exp \left[-i k_{1, z} z_{0,1}\right] \\
& \mathbf{E}_{0}^{\prime}(0) \exp \left[-i k_{0, z} z_{0,1}\right] \\
&=\boldsymbol{\Psi}_{0,1} \mathbf{E}_{0}(0) \exp \left[i k_{0, z} z_{0,1}\right] \\
& \quad+\boldsymbol{\Phi}_{1,0} \mathbf{E}_{1}^{\prime}(0) \exp \left[-i k_{1, z} z_{0,1}\right]
\end{aligned}
$$

$$
\begin{gathered}
\left(\begin{array}{cc}
\exp \left(-i k_{0, z} z_{0,1}\right) & 0 \\
0 & \exp \left(i k_{1, z} z_{0,1}\right)
\end{array}\right)\left(\begin{array}{c}
A_{0}^{\prime} \\
A_{1}
\end{array}\right)=\left(\begin{array}{cc}
\boldsymbol{\Psi}_{0,1} & \boldsymbol{\Phi}_{1,0} \\
\boldsymbol{\Phi}_{0,1} & \boldsymbol{\Psi}_{1,0}
\end{array}\right)\left(\begin{array}{cc}
\exp \left(i k_{0, z} z_{0,1}\right) & 0 \\
0 & \exp \left(-i k_{1, z} z_{0,1}\right)
\end{array}\right)\left(\begin{array}{c}
A_{0} \\
A_{1}^{\prime}
\end{array}\right) \\
\left(\begin{array}{c}
A_{0}^{\prime} \\
A_{1}
\end{array}\right)=\left(\begin{array}{cc}
\boldsymbol{\Psi}_{0,1} \exp \left(2 i k_{0, z} z_{0,1}\right) & \boldsymbol{\Phi}_{1,0} \exp \left(-i\left(k_{1, z}-k_{0, z}\right) z_{0,1}\right) \\
\boldsymbol{\Phi}_{0,1} \exp \left(i\left(k_{0, z}-k_{1, z}\right) z_{0,1}\right) & \boldsymbol{\Psi}_{1,0} \exp \left(-2 i k_{1, z} z_{0,1}\right)
\end{array}\right)\left(\begin{array}{c}
A_{0} \\
A_{1}^{\prime}
\end{array}\right)
\end{gathered}
$$

Then the amplitudes $A_{0}, A_{0}^{\prime}, A_{1}$, and $A_{1}^{\prime}$ are derived as follows:

$$
\begin{aligned}
& \mathbf{A}_{1} \exp \left(i k_{1, z} z_{0,1}\right) \\
& \quad=\boldsymbol{\Phi}_{0,1} \mathbf{A}_{0} \exp \left(i k_{0, z} z_{0,1}\right)+\Psi_{1,0} \mathbf{A}_{1}^{\prime} \exp \left(-i k_{1, z} z_{0,1}\right), \\
& \mathbf{A}_{0}^{\prime} \exp \left(-i k_{0, z} z_{0,1}\right) \\
& \quad=\Psi_{0,1} \mathbf{A}_{0} \exp \left(i k_{0, z} z_{0,1}\right)+\boldsymbol{\Phi}_{1,0} \mathbf{A}_{1}^{\prime} \exp \left(-i k_{1, z} z_{0,1}\right) .
\end{aligned}
$$

Matrix description of the relations is as follows:

\section{Then}

$$
\left(\begin{array}{c}
A_{0}^{\prime} \\
A_{1}
\end{array}\right)=\left(\begin{array}{cc}
\frac{k_{0, z}-k_{1, z}}{k_{0, z}+k_{1, z}} \exp \left(2 i k_{0, z} z_{0,1}\right) & \frac{2 k_{1, z}}{k_{0, z}+k_{1, z}} \exp \left(-i\left(k_{1, z}-k_{0, z}\right) z_{0,1}\right) \\
\frac{2 k_{0, z}}{k_{0, z}+k_{1, z}} \exp \left(i\left(k_{0, z}-k_{1, z}\right) z_{0,1}\right) & \frac{k_{1, z}-k_{0, z}}{k_{0, z}+k_{1, z}} \exp \left(-2 i k_{1, z} z_{0,1}\right)
\end{array}\right)\left(\begin{array}{c}
A_{0} \\
A_{1}^{\prime}
\end{array}\right)
$$

We take the average value of this matrix:

$$
\left(\begin{array}{c}
A_{0}^{\prime} \\
A_{1}
\end{array}\right)=\left(\begin{array}{cc}
\frac{k_{0, z}-k_{1, z}}{k_{0, z}+k_{1, z}}\left\langle\exp \left(2 i k_{0, z} z_{0,1}\right)\right\rangle & \frac{2 k_{1, z}}{k_{0, z}+k_{1, z}}\left\langle\exp \left(-i\left(k_{1, z}-k_{0, z}\right) z_{0,1}\right)\right\rangle \\
\frac{2 k_{0, z}}{k_{0, z}+k_{1, z}}\left\langle\exp \left(i\left(k_{0, z}-k_{1, z}\right) z_{0,1}\right)\right\rangle & \frac{k_{1, z}-k_{0, z}}{k_{0, z}+k_{1, z}}\left\langle\exp \left(-2 i k_{1, z} z_{0,1}\right)\right\rangle
\end{array}\right)\left(\begin{array}{c}
A_{0} \\
A_{1}^{\prime}
\end{array}\right)
$$

For Gaussian statistics of standard deviation value $\sigma$,

$$
\left(\begin{array}{c}
A_{0}^{\prime} \\
A_{1}
\end{array}\right)=\left(\begin{array}{cc}
\frac{k_{0, z}-k_{1, z}}{k_{0, z}+k_{1, z}} \exp \left(-2 k_{0, z}^{2} \sigma_{0,1}^{2}\right) & \frac{2 k_{1, z}}{k_{0, z}+k_{1, z}} \exp \left(-\frac{1}{2}\left(k_{0, z}-k_{1, z}\right)^{2} \sigma_{0,1}^{2}\right) \\
\frac{2 k_{0, z}}{k_{0, z}+k_{1, z}} \exp \left(-\frac{1}{2}\left(k_{0, z}-k_{1, z}\right)^{2} \sigma_{0,1}^{2}\right) & \frac{k_{1, z}-k_{0, z}}{k_{0, z}+k_{1, z}} \exp \left(-2 k^{2}{ }_{1, z} \sigma_{0,1}^{2}\right)
\end{array}\right)\left(\begin{array}{c}
A_{0} \\
A_{1}^{\prime}
\end{array}\right)
$$


Then the Fresnel reflection coefficients $\Psi^{\prime}$ are found as follows

$$
\begin{aligned}
& \Psi_{0,1}^{\prime}=\Psi_{0,1} \exp \left(-2 k_{0, z}^{2} \sigma_{0,1}^{2}\right), \\
& \Psi_{1,0}^{\prime}=\Psi_{1,0} \exp \left(-2 k_{1, z}^{2} \sigma_{0,1}^{2}\right),
\end{aligned}
$$

and the Fresnel refraction coefficients $\Phi^{\prime}$ are also produced similarly

$$
\begin{gathered}
\boldsymbol{\Phi}_{0,1}^{\prime}=\boldsymbol{\Phi}_{0,1} \exp \left(-\frac{1}{2}\left(k_{0, z}-k_{1, z}\right)^{2} \sigma_{0,1}^{2}\right), \\
\boldsymbol{\Phi}_{1,0}^{\prime}=\boldsymbol{\Phi}_{1,0} \exp \left(-\frac{1}{2}\left(k_{0, z}-k_{1, z}\right)^{2} \sigma_{0,1}^{2}\right), \\
\boldsymbol{\Phi}_{0,1}^{\prime} \boldsymbol{\Phi}_{1,0}^{\prime}-\boldsymbol{\Psi}_{0,1}^{\prime} \boldsymbol{\Psi}_{1,0}^{\prime} \\
=\frac{4 k_{0, z} k_{1, z}}{\left(k_{0, z}+k_{1, z}\right)^{2}} \exp \left(-\left(k_{0, z}-k_{1, z}\right)^{2} \sigma_{0,1}^{2}\right) \\
+\left(\frac{k_{1, z}-k_{0, z}}{k_{0, z}+k_{1, z}}\right)^{2} \exp \left(-2\left(k_{0, z}^{2}+k_{1, z}^{2}\right) \sigma_{0,1}^{2}\right)<1 .
\end{gathered}
$$

The modified Fresnel refraction coefficients $\Phi_{0,1}^{\prime}$ and $\Phi_{1,0}^{\prime}$ of (91) correspond to equation (1.115) on p.29 of Sakurai
[20]. The Fresnel refraction coefficients $\Phi^{\prime}$ derived by this method are reduced and could be used to calculate the reflectivity from rough surface and interfaces. Accordingly, we calculated the reflectivity using these derived Fresnel refraction coefficients. However, the numerical results of this calculation did not agree with the experimental results when the angle of incidence smaller than the total reflection critical angle. In trying to account for the reason for this disagreement, it should be noticed that our present approach to constructing the reduced reflection coefficient $\Psi_{0,1}^{\prime}$ term does not include any reference to the refractive index of the medium. Further, X-rays that penetrate an interface reflect from the interface below, and penetrate the former interface again without fail. Therefore, the refraction coefficient $\Phi_{0,1}^{\prime}$ and $\Phi_{1,0}^{\prime}$ should not be separately treated.

3.6. A New Formula for the Reflectivity for Rough Multilayer Surface. Once again we consider process by which we derive the average value of the matrix. When the $z$ position of the interface of 0th layer and 1th layer $z_{0,1}$ fluctuates vertically as a function of the lateral position because of the interface roughness, the relations between the amplitudes $A_{0}, A_{0}^{\prime}, A_{1}$, and $A_{1}^{\prime}$ are shown by the use of the Fresnel coefficient tensor $\Phi$ for refraction and the Fresnel coefficient tensor $\Psi$ for reflection as follows:

$$
\begin{aligned}
& \left(\begin{array}{cc}
\exp \left(-i k_{0, z} z_{0,1}\right) & 0 \\
0 & \exp \left(i k_{1, z} z_{0,1}\right)
\end{array}\right)\left(\begin{array}{l}
A_{0}^{\prime} \\
A_{1}
\end{array}\right)=\left(\begin{array}{cc}
\boldsymbol{\Psi}_{0,1} & \boldsymbol{\Phi}_{1,0} \\
\boldsymbol{\Phi}_{0,1} & \boldsymbol{\Psi}_{1,0}
\end{array}\right)\left(\begin{array}{cc}
\exp \left(i k_{0, z} z_{0,1}\right) & 0 \\
0 & \exp \left(-i k_{1, z} z_{0,1}\right)
\end{array}\right)\left(\begin{array}{l}
A_{0} \\
A_{1}^{\prime}
\end{array}\right) \\
& \left(\begin{array}{c}
A_{0}^{\prime} \\
A_{1}
\end{array}\right)=\frac{1}{\exp \left(-i k_{0, z} z_{0,1}\right) \exp \left(i k_{1, z} z_{0,1}\right)}\left(\begin{array}{cc}
\exp \left(i k_{1, z} z_{0,1}\right) & 0 \\
0 & \exp \left(-i k_{0, z} z_{0,1}\right)
\end{array}\right)\left(\begin{array}{ll}
\boldsymbol{\Psi}_{0,1} & \boldsymbol{\Phi}_{1,0} \\
\boldsymbol{\Phi}_{0,1} & \boldsymbol{\Psi}_{1,0}
\end{array}\right) \\
& \times\left(\begin{array}{cc}
\exp \left(i k_{0, z} z_{0,1}\right) & 0 \\
0 & \exp \left(-i k_{1, z} z_{0,1}\right)
\end{array}\right)\left(\begin{array}{c}
A_{0} \\
A_{1}^{\prime}
\end{array}\right) \\
& \left(\begin{array}{c}
A_{0}^{\prime} \\
A_{1}
\end{array}\right)=\frac{1}{\exp \left(-i k_{0, z} z_{0,1}\right) \exp \left(i k_{1, z} z_{0,1}\right)} \\
& \times\left(\begin{array}{cc}
\exp \left(i k_{1, z} z_{0,1}\right) \Psi_{0,1} \exp \left(i k_{0, z} z_{0,1}\right) & \exp \left(i k_{1, z} z_{0,1}\right) \Phi_{1,0} \exp \left(-i k_{1, z} z_{0,1}\right) \\
\exp \left(-i k_{0, z} z_{0,1}\right) \Phi_{0,1} \exp \left(i k_{0, z} z_{0,1}\right) & \exp \left(-i k_{0, z} z_{0,1}\right) \Psi_{1,0} \exp \left(-i k_{1, z} z_{0,1}\right)
\end{array}\right)\left(\begin{array}{c}
A_{0} \\
A_{1}^{\prime}
\end{array}\right), \\
& \left(\begin{array}{c}
A_{0}^{\prime} \\
A_{1}
\end{array}\right)=\frac{1}{\exp \left(-i k_{0, z} z_{0,1}\right) \exp \left(i k_{1, z} z_{0,1}\right)} \\
& \times\left(\begin{array}{cc}
\boldsymbol{\Psi}_{0,1} \exp \left(i\left(k_{1, z}+k_{0, z}\right) z_{0,1}\right) & \boldsymbol{\Phi}_{1,0} \exp \left(i\left(k_{1, z}-k_{1, z}\right) z_{0,1}\right) \\
\boldsymbol{\Phi}_{0,1} \exp \left(i\left(-k_{0, z}+k_{0, z}\right) z_{0,1}\right) & \boldsymbol{\Psi}_{1,0} \exp \left(i\left(-k_{0, z}-k_{1, z}\right) z_{0,1}\right)
\end{array}\right)\left(\begin{array}{c}
A_{0} \\
A_{1}^{\prime}
\end{array}\right), \\
& \left(\begin{array}{c}
A_{0}^{\prime} \\
A_{1}
\end{array}\right)=\left(\begin{array}{cc}
\Psi_{0,1} \frac{\exp \left(i\left(k_{1, z}+k_{0, z}\right) z_{0,1}\right)}{\exp \left(i\left(-k_{0, z}+k_{1, z}\right) z_{0,1}\right)} & \boldsymbol{\Phi}_{1,0} \exp \left(i\left(k_{0, z}-k_{1, z}\right) z_{0,1}\right) \\
\boldsymbol{\Phi}_{0,1} \exp \left(i\left(-k_{1, z}+k_{0, z}\right) z_{0,1}\right) & \Psi_{1,0} \frac{\exp \left(i\left(-k_{0, z}-k_{1, z}\right) z_{0,1}\right)}{\exp \left(i\left(-k_{0, z}+k_{1, z}\right) z_{0,1}\right)}
\end{array}\right)\left(\begin{array}{l}
A_{0} \\
A_{1}^{\prime}
\end{array}\right) .
\end{aligned}
$$


Again, we take the average value of this matrix:

$$
\left(\begin{array}{c}
A_{0}^{\prime} \\
A_{1}
\end{array}\right)=\left(\begin{array}{cc}
\Psi_{0,1} \frac{\left\langle\exp \left(i\left(k_{1, z}+k_{0, z}\right) z_{0,1}\right)\right\rangle}{\left\langle\exp \left(i\left(-k_{0, z}+k_{1, z}\right) z_{0,1}\right)\right\rangle} & \boldsymbol{\Phi}_{1,0}\left\langle\exp \left(i\left(k_{0, z}-k_{1, z}\right) z_{0,1}\right)\right\rangle \\
\boldsymbol{\Phi}_{0,1}\left\langle\exp \left(i\left(-k_{1, z}+k_{0, z}\right) z_{0,1}\right)\right\rangle & \boldsymbol{\Psi}_{1,0} \frac{\left\langle\exp \left(i\left(-k_{0, z}-k_{1, z}\right) z_{0,1}\right)\right\rangle}{\left\langle\exp \left(i\left(-k_{0, z}+k_{1, z}\right) z_{0,1}\right)\right\rangle}
\end{array}\right)\left(\begin{array}{c}
A_{0} \\
A_{1}^{\prime}
\end{array}\right)
$$

For Gaussian statistics of standard deviation value $\sigma$, the Fresnel reflection coefficient $\Psi^{\prime}$ are as follows:

$$
\begin{aligned}
\boldsymbol{\Psi}_{0,1}^{\prime} & =\Psi_{0,1} \frac{\left\langle\exp \left(i\left(k_{1, z}+k_{0, z}\right) z_{0,1}\right)\right\rangle}{\left\langle\exp \left(i\left(-k_{0, z}+k_{1, z}\right) z_{0,1}\right)\right\rangle} \\
& =\Psi_{0,1} \frac{\exp \left(-(1 / 2)\left(k_{0, z}+k_{1, z}\right)^{2} \sigma_{0,1}^{2}\right)}{\exp \left(-(1 / 2)\left(k_{0, z}-k_{1, z}\right)^{2} \sigma_{0,1}^{2}\right)} \\
& =\Psi_{0,1} \exp \left(-2 k_{0, z} k_{1, z} \sigma_{0,1}^{2}\right), \\
\boldsymbol{\Psi}_{1,0}^{\prime} & =\Psi_{1,0} \frac{\left\langle\exp \left(i\left(-k_{0, z}-k_{1, z}\right) z_{0,1}\right)\right\rangle}{\left\langle\exp \left(i\left(-k_{0, z}+k_{1, z}\right) z_{0,1}\right)\right\rangle} \\
& =\Psi_{1,0} \frac{\exp \left(-(1 / 2)\left(-k_{0, z}-k_{1, z}\right)^{2} \sigma_{0,1}^{2}\right)}{\exp \left(-(1 / 2)\left(k_{1, z}-k_{0, z}\right)^{2} \sigma_{0,1}^{2}\right)} \\
& =\Psi_{1,0} \exp \left(-2 k_{0, z} k_{1, z} \sigma_{0,1}^{2}\right) .
\end{aligned}
$$

Because X-rays that penetrate an interface reflect from the interface below and penetrate former interface again without fail, it is necessary to treat the refraction coefficients $\Phi_{0,1}^{\prime}$ and $\Phi_{1,0}^{\prime}$ collectively:

$$
\begin{aligned}
\boldsymbol{\Phi}_{0,1}^{\prime} \boldsymbol{\Phi}_{1,0}^{\prime}=\langle & \boldsymbol{\Phi}_{0,1} \exp \left(i\left(-k_{1, z}+k_{0, z}\right) z_{0,1}\right) \\
& \left.\times \boldsymbol{\Phi}_{1,0} \exp \left(i\left(k_{0, z}-k_{1, z}\right) z_{0,1}\right)\right\rangle
\end{aligned}
$$

$$
\begin{gathered}
\left(\begin{array}{c}
A_{0}^{\prime} \\
A_{1}
\end{array}\right)=\left(\begin{array}{cc}
\boldsymbol{\Psi}_{0,1} \exp \left(-2 k_{0, z} k_{1, z} \sigma_{0,1}^{2}\right) & \boldsymbol{\Phi}_{1,0} \exp \left(-\left(k_{0, z}-k_{1, z}\right)^{2} \sigma_{0,1}^{2}\right) \\
\boldsymbol{\Phi}_{0,1} \exp \left(-\left(k_{0, z}-k_{1, z}\right)^{2} \sigma_{0,1}^{2}\right) & \boldsymbol{\Psi}_{1,0} \exp \left(-2 k_{0, z} k_{1, z} \sigma_{0,1}^{2}\right)
\end{array}\right)\left(\begin{array}{c}
A_{0} \\
A_{1}^{\prime}
\end{array}\right), \\
\left(\begin{array}{c}
A_{0}^{\prime} \\
A_{1}
\end{array}\right)=\left(\begin{array}{cc}
\frac{k_{0, z}-k_{1, z}}{k_{0, z}+k_{1, z}} \exp \left(-2 k_{0, z} k_{1, z} \sigma_{0,1}^{2}\right) & \frac{2 k_{1, z}}{k_{0, z}+k_{1, z}} \exp \left(-\left(k_{0, z}-k_{1, z}\right)^{2} \sigma_{0,1}^{2}\right) \\
\frac{2 k_{0, z}}{k_{0, z}+k_{1, z}} \exp \left(-\left(k_{0, z}-k_{1, z}\right)^{2} \sigma_{0,1}^{2}\right) & \frac{k_{1, z}-k_{0, z}}{k_{0, z}+k_{1, z}} \exp \left(-2 k_{0, z} k_{1, z} \sigma_{0,1}^{2}\right)
\end{array}\right)\left(\begin{array}{c}
A_{0} \\
A_{1}^{\prime}
\end{array}\right), \\
\boldsymbol{\Phi}_{0,1}^{\prime} \boldsymbol{\Phi}_{1,0}^{\prime}-\Psi_{0,1}^{\prime} \Psi_{1,0}^{\prime}=\frac{4 k_{0, z} k_{1, z}}{\left(k_{0, z}+k_{1, z}\right)^{2}} \exp \left(-2\left(k_{0, z}-k_{1, z}\right)^{2} \sigma_{0,1}^{2}\right)+\left(\frac{k_{1, z}-k_{0, z}}{k_{0, z}+k_{1, z}}\right)^{2} \exp \left(-4 k_{0, z} k_{1, z} \sigma_{0,1}^{2}\right)<1 .
\end{gathered}
$$




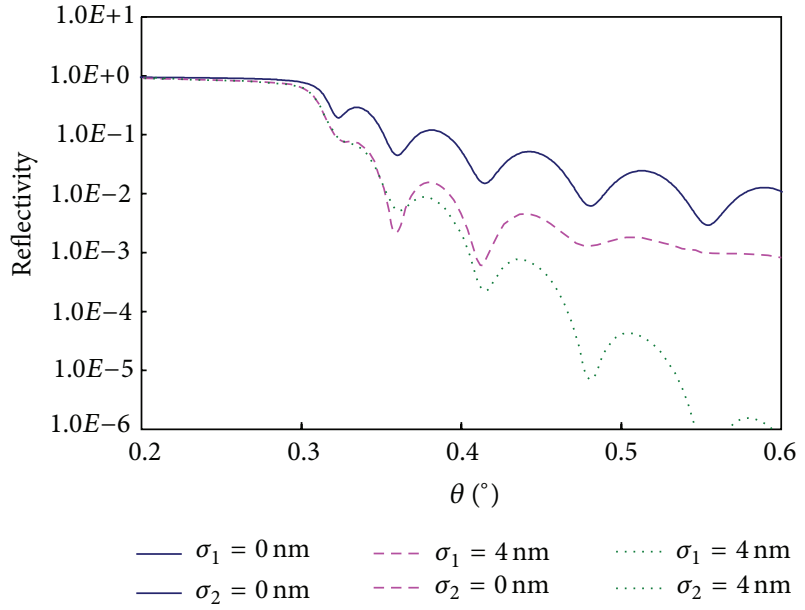

Figure 10: New calculated reflectivities from a GaAs layer with a thickness of $48 \mathrm{~nm}$ on a Si substrate. The line is for a flat surface and a flat interface. The dashed curve is for a surface roughness $\sigma_{1}$ of $4 \mathrm{~nm}$ and with a flat interface, while the dotted curve is for a surface roughness $\sigma_{1}$ of $4 \mathrm{~nm}$ and interface roughness $\sigma_{2}$ of $4 \mathrm{~nm}$.

The Fresnel refraction coefficients $\Phi^{\prime}$ derived by this method are reduced and can be used to calculate the reflectivity from rough surface and interface. Therefore, we calculate the reflectivity using these newly derived Fresnel coefficients in an accurate reflectivity equation of $R_{j-1, j}$ and $R_{j, j+1}$ as follows:

$$
\begin{aligned}
& \mathbf{R}_{j-1, j} \\
& =\frac{\Psi_{j-1, j}^{\prime}+\left(\boldsymbol{\Phi}_{j-1, j}^{\prime} \boldsymbol{\Phi}_{j, j-1}^{\prime}-\Psi_{j-1, j}^{\prime} \Psi_{j, j-1}^{\prime}\right) R_{j, j+1}}{1-\Psi_{j, j-1}^{\prime} \mathbf{R}_{j, j+1}} \\
& \quad \times \exp \left(2 i k_{j-1, z} h_{j-1}\right) .
\end{aligned}
$$

Based on the previous considerations, we again calculated the $\mathrm{X}$-ray reflectivity for the GaAs/Si system but now considered the effect of attenuation in the refracted X-rays by diffuse scattering resulting from surface roughness. The results are shown as the dashed line in Figure 10 for a surface roughness of $4 \mathrm{~nm}$ and flat interface, and the dotted line shows the calculated result in the case that the surface and interface both have an rms roughness of $4 \mathrm{~nm}$.

The ratio of the oscillation amplitude to the size of the reflectivity in the reflectivity curve (dot) in Figure 10 is smaller than that of the reflectivity curve Figure 7 . In the reflectivity curve (dashed line), the very large amplitude of the oscillation near an angle of incidence of $0.36^{\circ}$ in Figure 7 has disappeared in Figure 10. These results are now physically reasonable. All the strange results seen in Figure 7 have disappeared in Figure 10. It seems natural that the effect of interference does decrease at a rough surface and interface, because the amount of coherent X-rays should reduce due to diffuse scattering.

Figure 11 shows the new calculated reflectivity for surface roughnesses of $3.5 \mathrm{~nm}, 4 \mathrm{~nm}$, and $4.5 \mathrm{~nm}$ and with a flat interface. At all angles of incidence, the reflectivity of

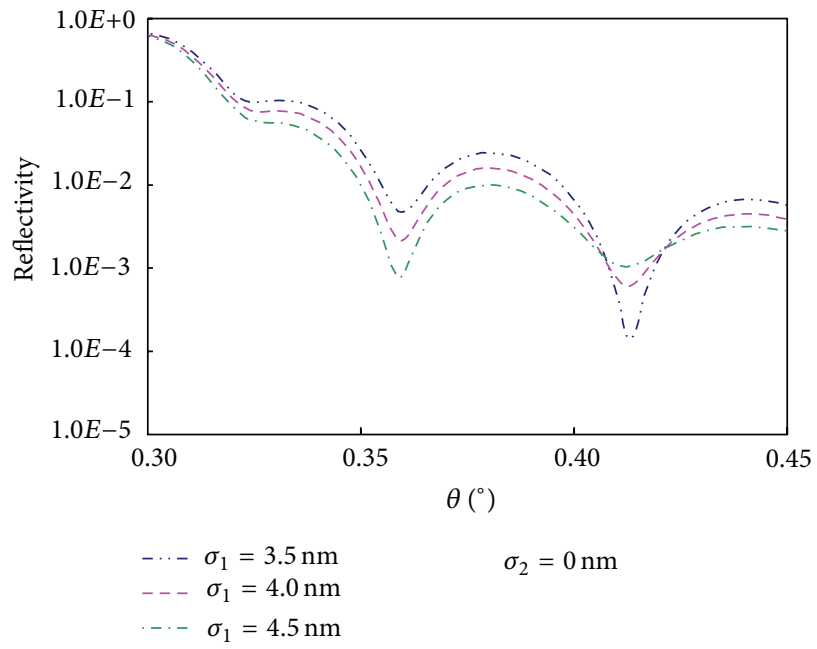

FIGURE 11: New calculated reflectivity from a GaAs layer with a thickness of $48 \mathrm{~nm}$ on a Si substrate. In the calculation, the interface roughness $\sigma_{2}$ is $0 \mathrm{~nm}$. Three calculated results for a GaAs surface with roughness $\sigma_{1}$ of $3.5 \mathrm{~nm}, 4 \mathrm{~nm}$, and $4.5 \mathrm{~nm}$ are shown.

the surface roughness of $4 \mathrm{~nm}$ is near the mean value of the reflectivity of the surface roughness of $3.5 \mathrm{~nm}$ and the reflectivity of the surface roughness of $4.5 \mathrm{~nm}$. This result is physically reasonable, because the value of the roughness is the standard deviation value of various amplitudes of rough surface. However, it was difficult to match the numerical result of X-ray reflectivity to the results of TEM observation.

Next, we again calculated the X-ray reflectivity for the W/Si system but now considered the effect of attenuation in the refracted $\mathrm{X}$ rays by diffuse scattering resulting from surface roughness. However, the reduced refraction coefficient in prior work varies [13-19]. Then about the reduced refraction coefficient, reduction as same as reflection coefficient was applied now. Figure 12 shows the calculated results with the use of improved X-ray reflectivity formalism. In the reflectivity curve from a surface with an rms surface roughness of $0.3 \mathrm{~nm}$ (dashed line), the amplitude of the oscillation in Figure 9 has reduced in Figure 12. These results are now physically reasonable. The strange results seen in Figure 9 have disappeared in Figure 12. It seems natural that the effect of interference does decrease at a rough surface and interface, because the amount of coherent $\mathrm{X}$ rays should reduce due to diffuse scattering.

\section{Summary}

In this review, we investigated the fact that the calculated result of the X-ray reflectivity based on Parratt formalism [1] with the effect of the roughness incorporated by the theory of Nevot-Croce [2] shows a strange phenomenon in which the amplitude of the oscillation due to the interference effects increases in the case of the rougher surface. The X-ray reflectivity calculation based on Parratt formalism [1] with the effect of the roughness incorporated by the theory of Nevot-Croce [2] shows as in (48), with the reduced Fresnel 


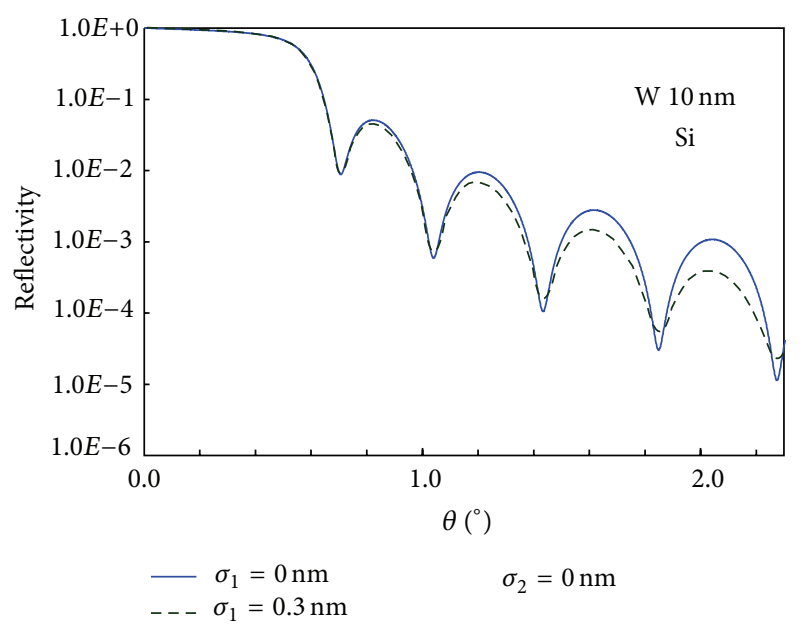

FIGURE 12: X-ray reflectivity from a silicon wafer covered with a thin $(10 \mathrm{~nm})$ tungsten film calculated by the new calculation that considered diffuse scattering. Solid line shows the case of a flat surface. Dashed line shows the case of a surface with an rms surface roughness of $0.3 \mathrm{~nm}$.

reflection coefficient $\Psi^{\prime}$ being as shown in (47). However, the relationship between the reflection coefficients $R_{j-1, j}$ and $R_{j, j+1}$ was originally derived as in (51). Here, the following conditional relations between the Fresnel coefficient for reflection and refraction are relevant to (51); see (52) and (53). In these condition, the Fresnel coefficients for refraction at the rough interface are derived using the Fresnel reflection coefficient $\Psi$ as shown in (57).

Therefore, the Fresnel coefficients for refraction at the rough interface are necessarily larger than the Fresnel coefficient for refraction at the flat interface. The resulting increase in the transmission coefficient completely overpowers any decrease in the value of the reflection coefficient. These coefficients for refraction obviously contain a mistake because the penetration of X-rays should decrease at a rough interface because of diffuse scattering. We propose that the unnatural results in the previous calculation of the X-ray reflectivity originate from the fact that diffuse scattering was not considered. We found that the strange result originates in the currently used equation due to a serious mistake where the Fresnel refraction coefficient in the reflectivity equation is increased at a rough interface. The increase in the transmission coefficient completely overpowers any decrease in the value of the reflection coefficient because of a lack of consideration of diffuse scattering. The mistake in Nevot and Croce's treatment originates in the fact that the modified Fresnel coefficients were calculated based on the theory, which contains the X-ray energy conservation rule at the surface and interface. In their discussion, the transmission coefficient was replaced by the reflection coefficient so as to conserve energy, and so diffuse scattering was ignored at the rough interface. It is meaningless to try to precisely match the numerical result based on a wrong calculating formula even to details of the reflectivity profile of the experimental result. Thus, because Nevot and Croce's treatment of the Parratt formalism contains a fundamental mistake regardless of the size of roughness, results based on this approach are not correct.

We have developed a new formalism that corrects this mistake, producing more accurate estimates of the X-ray reflectivity for systems having surface and interfacial roughness, taking into account the effect of roughness-induced diffuse scattering.

The new, accurate formalism is completely described in detail. The X-ray reflectivity $R$ of a multilayer thin film material consisting of $N$ layers is derived by the use of accurate reflectivity equations for $R_{j-1, j}$ and $R_{j, j+1}$ as the following:

$$
\begin{gathered}
R=\left|R_{0,1}\right|^{2}, \\
R_{j-1, j}=\frac{\Psi_{j-1, j}+\left(\Phi_{j-1, j} \Phi_{j, j-1}-\Psi_{j-1, j} \Psi_{j, j-1}\right) R_{j, j+1}}{1-\Psi_{j, j-1} R_{j, j+1}} \\
\times \exp \left(2 i k_{j-1, z} h_{j-1}\right), \\
R_{N, N+1}=0 .
\end{gathered}
$$

Here, the refractive index of the $j$ th layer $n_{j}=1-\delta_{j}-$ $i \beta_{j}, n_{0}=1$, the $z$-direction component of the wave vector of the $j$ th layer $k_{j, z}=k \sqrt{n_{j}^{2}-\cos ^{2} \theta}, k=2 \pi / \lambda$, $\lambda$; wave length, $\theta$; glancing angle of incidence, a $N$-layer multilayer system with a $j$ th layer of thickness of $h_{j}$ and $j-1, j$ th interface roughness of $\sigma_{j-1, j}, k_{j, z}$ is the $z$ component of the wave vector in the $j$ th layer, and $\Psi_{j-1, j}$ and $\Phi_{j-1, j}$ are the Fresnel coefficients for reflection and refraction, respectively, at the interface between the $(j-1)$ th layer and the $j$ th layer. Although formula for $\Psi_{j-1, j}$ is well known

$$
\begin{gathered}
\Psi_{j-1, j}=\frac{k_{j-1, z}-k_{j, z}}{k_{j-1, z}+k_{j, z}} \exp \left(-2 k_{j-1, z} k_{j, z} \sigma_{j-1, j}^{2}\right), \\
\Psi_{j, j-1}=-\Psi_{j-1, j},
\end{gathered}
$$

where $\sigma_{j-1, j}$ is the interface roughness between $(j-1)$ th and $j$ th layers, an accurate analytical formula for $\Phi_{j-1, j}$ including the effect of the interface roughness is not available. There are several approximations proposed so far and all these results can be written as

$$
\begin{gathered}
\Phi_{j-1, j}=\frac{2 k_{j-1, z}}{k_{j-1, z}+k_{j, z}} \\
\times \exp \left\{-\left[C_{1}\left(k_{j-1, z}-k_{j, z}\right)^{2}+C_{2} k_{j-1, z} k_{j, z}\right] \sigma_{0,1}^{2}\right\}, \\
\Phi_{j, j-1}=\Phi_{j-1, j} \frac{k_{j, z}}{k_{j-1, z}},
\end{gathered}
$$

where parameters $C_{1}, C_{2}$ depend on the proposed approximation. $C_{1}=2$ and $C_{2}=0$ is the most appropriate approximation [23]. 


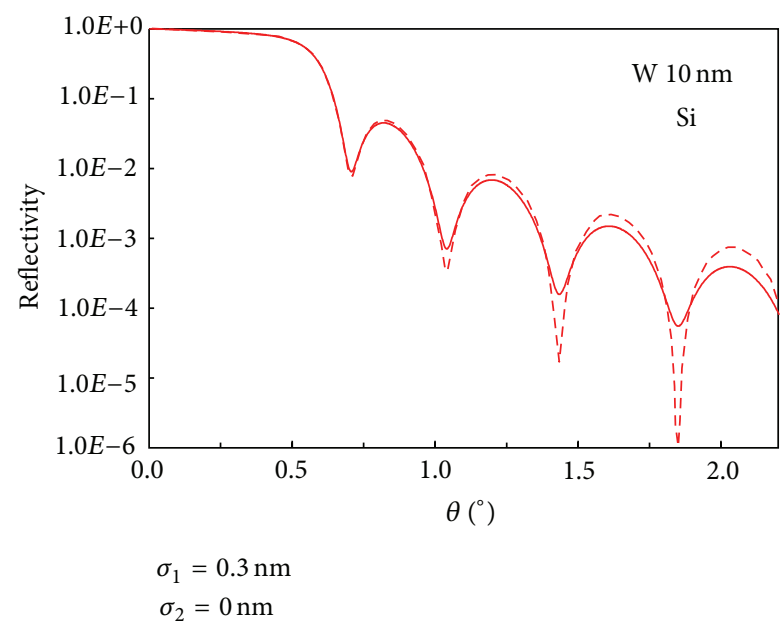

FIGURE 13: X-ray reflectivity from a silicon wafer covered with a thin $(10 \mathrm{~nm})$ tungsten film with an rms surface roughness of $0.3 \mathrm{~nm}$. Dashed line shows the calculated result by the theory in use prior to this work. Solid line shows the calculated result by the new calculation that considered the reduction in the sum intensity of reflective X-ray and refractive X-ray by diffuse scattering.

The penetration of X-rays should decrease at a rough interface because of diffuse scattering. Therefore, the identity equation for the Fresnel coefficients become

$$
\begin{aligned}
& \Phi_{j-1, j}^{\prime} \Phi_{j, j-1}^{\prime}-\Psi_{j-1, j}^{\prime} \Psi_{j, j-1}^{\prime} \\
& =\Phi_{j-1, j}^{\prime} \Phi_{j, j-1}^{\prime}+\Psi_{j-1, j}^{\prime 2} \\
& =1-D^{2}<1,
\end{aligned}
$$

where $D^{2}$ is a decrease due to diffuse scattering. Then, in the calculation of X-ray reflectivity when there is roughening at the surface or the interface, the Fresnel transmission coefficient $\Phi^{\prime}$ should be used for the reduced coefficient.

Figure 13 shows the reflectivity from a tungsten-covered silicon wafer with an rms surface roughness of $0.3 \mathrm{~nm}$. Dashed line shows the calculated result by (48) based on Parratt formalism with the effect of the roughness incorporated by the theory of Nevot-Croce in use prior to this work. The ratio of the oscillation amplitude to the value of the reflectivity from a surface with an rms surface roughness of $0.3 \mathrm{~nm}$ does not decrease near an angle of incidence of $1.8^{\circ}$ but increases than the reflectivity from a flat surface in Figure 9. This result is strange and not reasonable. Next, we again calculated the $\mathrm{X}$ ray reflectivity for the $\mathrm{W} / \mathrm{Si}$ system, but now considered the effect of attenuation in the refracted $\mathrm{X}$ rays and the reduction in the sum intensity of reflective X-ray and refractive Xray by diffuse scattering. Solid line shows the calculated results with the use of improved X-ray reflectivity formalism. In the reflectivity curve, the amplitude of the oscillation is smallerthan that of the reflectivity from a flat surface in Figure 12. These results are now physically reasonable. The strange results seen in the previous calculation have disappeared. It seems natural that the effect of interference does decrease at a rough surface and interface, because the amount of coherent $\mathrm{X}$ rays should reduce due to diffuse scattering.

The reflectivity calculated with this new, accurate formalism (105) gives a physically reasonable result. The use of this equation resolves the strange numerical results that occurred in the previous calculations that neglected diffuse scattering and is expected that buried interface structure can now be analyzed more accurately.

\section{References}

[1] L. G. Parratt, "Surface studies of solids by ttal reflection of Xrays," Physical Review, vol. 95, no. 2, pp. 359-369, 1954.

[2] L. Nevot and P. Croce, "Caractérisation des surfaces par réflexion rasante de rayons X. Application à l'étude du polissage de quelques verres silicates," Revue de Physique Appliquée, vol. 15, no. 3, pp. 761-779, 1980.

[3] W. C. Marra, P. Eisenberger, and A. Y. Cho, "X-ray totalexternal-reflection-Bragg diffraction: a structural study of the GaAs-Al interface," Journal of Applied Physics, vol. 50, no. 11, pp. 6927-6933, 1979.

[4] G. H. Vineyard, "Grazing-incidence diffraction and the distorted-wave approximation for the study of surfaces," Physical Review B, vol. 26, no. 8, pp. 4146-4159, 1982.

[5] I. K. Robinson, "Crystal truncation rods and surface roughness Crystal truncation rods and surface roughness," Physical Review $B$, vol. 33, no. 6, pp. 3830-3836, 1986.

[6] K. Sakurai and A. Iida, "Fourier analysis of interference structure in X-ray specular reflection from thin films " Japanese Journal of Applied Physics, vol. 31, pp. L113-L115, 1992.

[7] Y. Sakaida, S. Harada, and K. Tanaka, "Estimation of residual stress distribution of ground ceramics by glancing incidence X-ray diffraction technique," Journal of the Society of Materials Science, vol. 42, no. 477, pp. 641-647, 1993.

[8] Y. Fujii, T. Nakayama, and K. Yoshida, "Roughness estimation of polycrystalline iron surface under high temperature by small glancing angle x-ray scattering," ISIJ International, vol. 44, no. 9, pp. 1549-1553, 2004.

[9] Y. Fujii, T. Komai, and K. Ikeda, "Depth profiling of polycrystalline layers under a surface using $\mathrm{x}$-ray diffraction at small glancing angle of incidence," Surface and Interface Analysis, vol. 37, no. 2, pp. 190-193, 2005.

[10] Y. Fujii, E. Yanase, and K. Arai, "Depth profiling of the strain distribution in the surface layer using X-ray diffraction at small glancing angle of incidence," Applied Surface Science, vol. 244, no. 1-4, pp. 230-234, 2005.

[11] Y. Fujii and T. Nakayama, "Estimation of crystal grain size near surface using X-ray scattering at small glancing angles of incidence," Surface and Interface Analysis, vol. 38, no. 12-13, pp. 1679-1682, 2006.

[12] Y. Fujii, E. Yanase, and K. Nishio, "A proposal of depth profile analysis method of strain distribution in surface layer using $\mathrm{X}$ ray diffraction at small glancing angles of incidence," Journal of Physics, vol. 83, Article ID 012008, 2007.

[13] Y. Fujii, "Simple method of surface characterization by X-ray scattering at small glancing angle of incidence using a compact UHV X-ray diffractometer," Surface and Interface Analysis, vol. 40, no. 13, pp. 1722-1724, 2008.

[14] V. Holy, U. Pietsch, and T. Baumbach, Eds., High-Resolution XRay Scattering from Thin Films and Multilayers, Springer, Berlin, German, 1999. 
[15] J. Daillant and A. Gibaud, Eds., X-Ray and Neutron Reflectivity, Principles and Applications, Springer, Berlin, German, 1999.

[16] S. K. Sinha, E. B. Sirota, S. Garoff, and H. B. Stanley, "X-ray and neutron scattering from rough surfaces," Physical Review B, vol. 38, no. 4, pp. 2297-2311, 1988.

[17] V. Holy, J. Kubena, I. Ohlidal, K. Lischka, and W. Plotz, "X-ray reflection from rough layered systems," Physical Review B, vol. 47, no. 23, pp. 15896-15903, 1993.

[18] C. Slater and N. H. Frank, Electromagnetism, McGraw-Hill, New York, NY, USA, 1947.

[19] B. Vidal and P. Vincent, "Metallic multilayers fox x rays using classical thin-film theory," Applied Optics, vol. 23, no. 11, pp. 1794-1801, 1984.

[20] D. K. G. Boer, "X-ray reflection and transmission by rough surfaces," Physical Review B, vol. 51, no. 8, pp. 5297-5305, 1995.

[21] K. Sakurai, Ed., Introduction to X-Ray Reflectivity, Kodansha Scientific, Tokyo, Japan, 2009.

[22] Y. Fujii, "Influence of surface roughness on near-surface depth analysis from X-ray reflectivity measurements," Surface and Interface Analysis, vol. 42, no. 10-11, pp. 1642-1645, 2010.

[23] Y. Fujii, "Studies of nanostructures formed in T-10 tokamak," Materials Science and Engineering, vol. 24, Article ID 012008, 9 pages, 2011.

[24] Y. Fujii, "Improved x-ray reflectivity calculations for rough surfaces and interfaces," Materials Science and Engineering, vol. 24, Article ID 012009, 21 pages, 2011. 

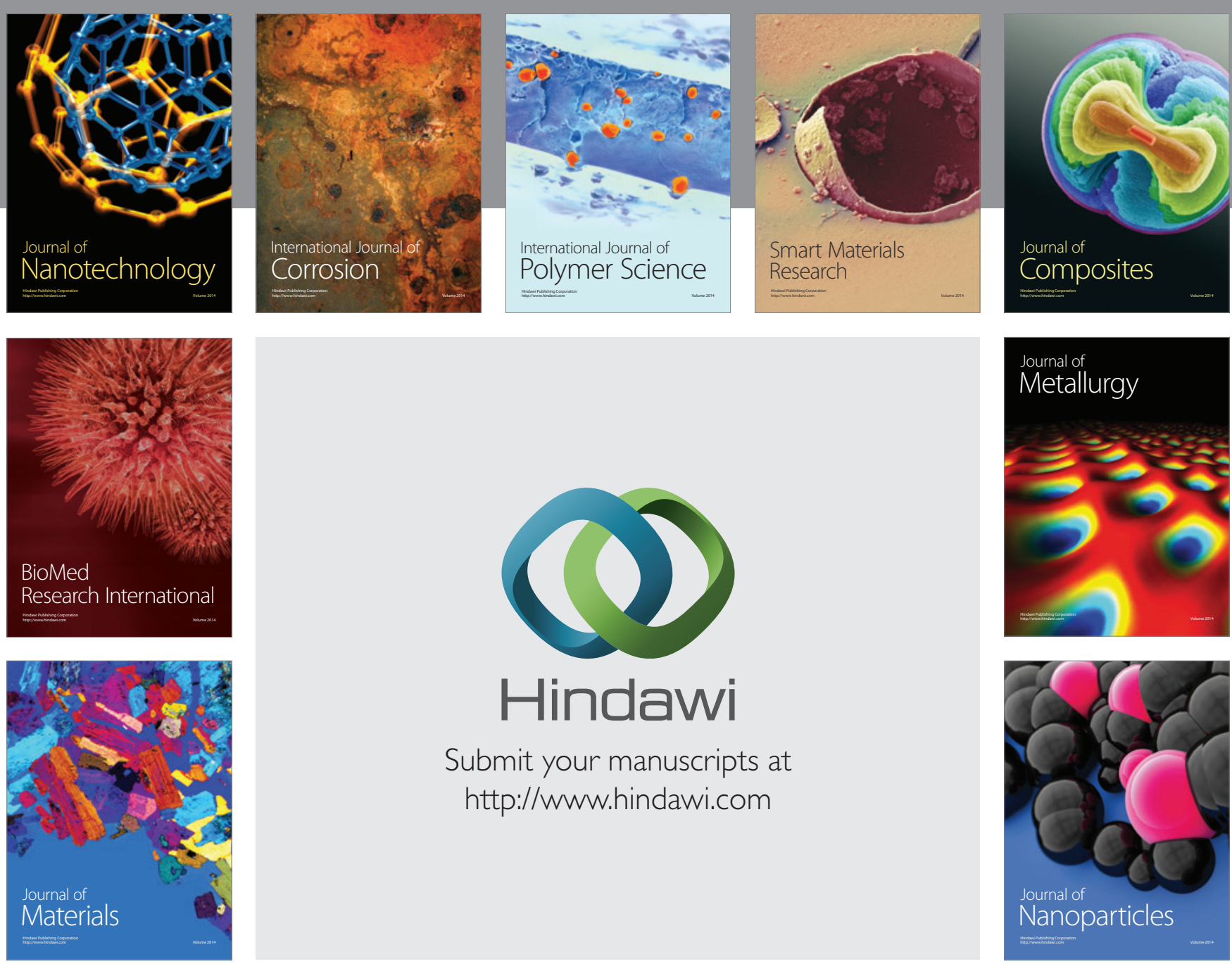

Submit your manuscripts at http://www.hindawi.com
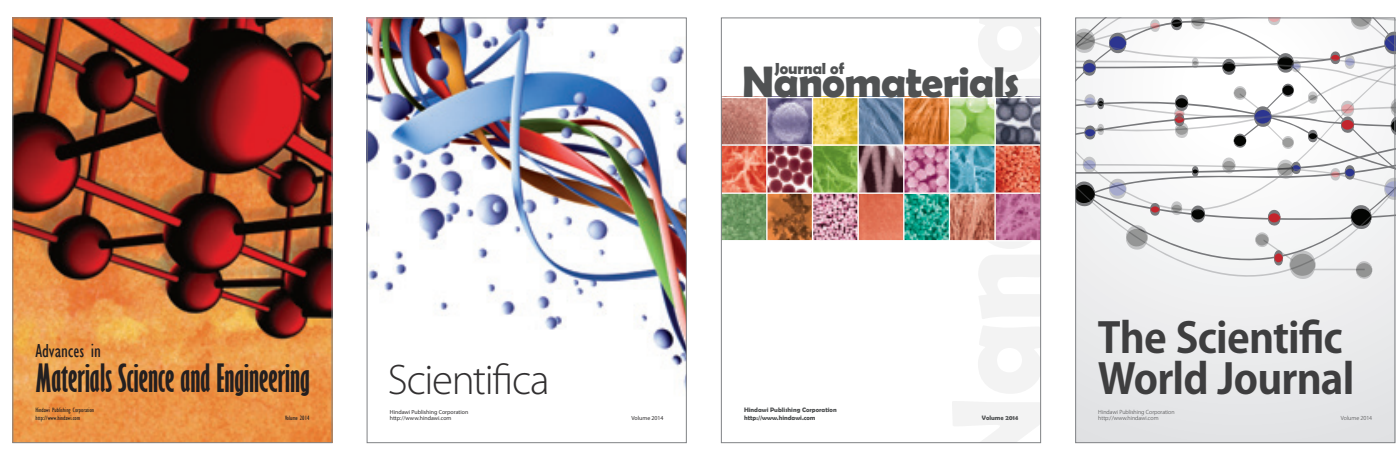

\section{The Scientific World Journal}
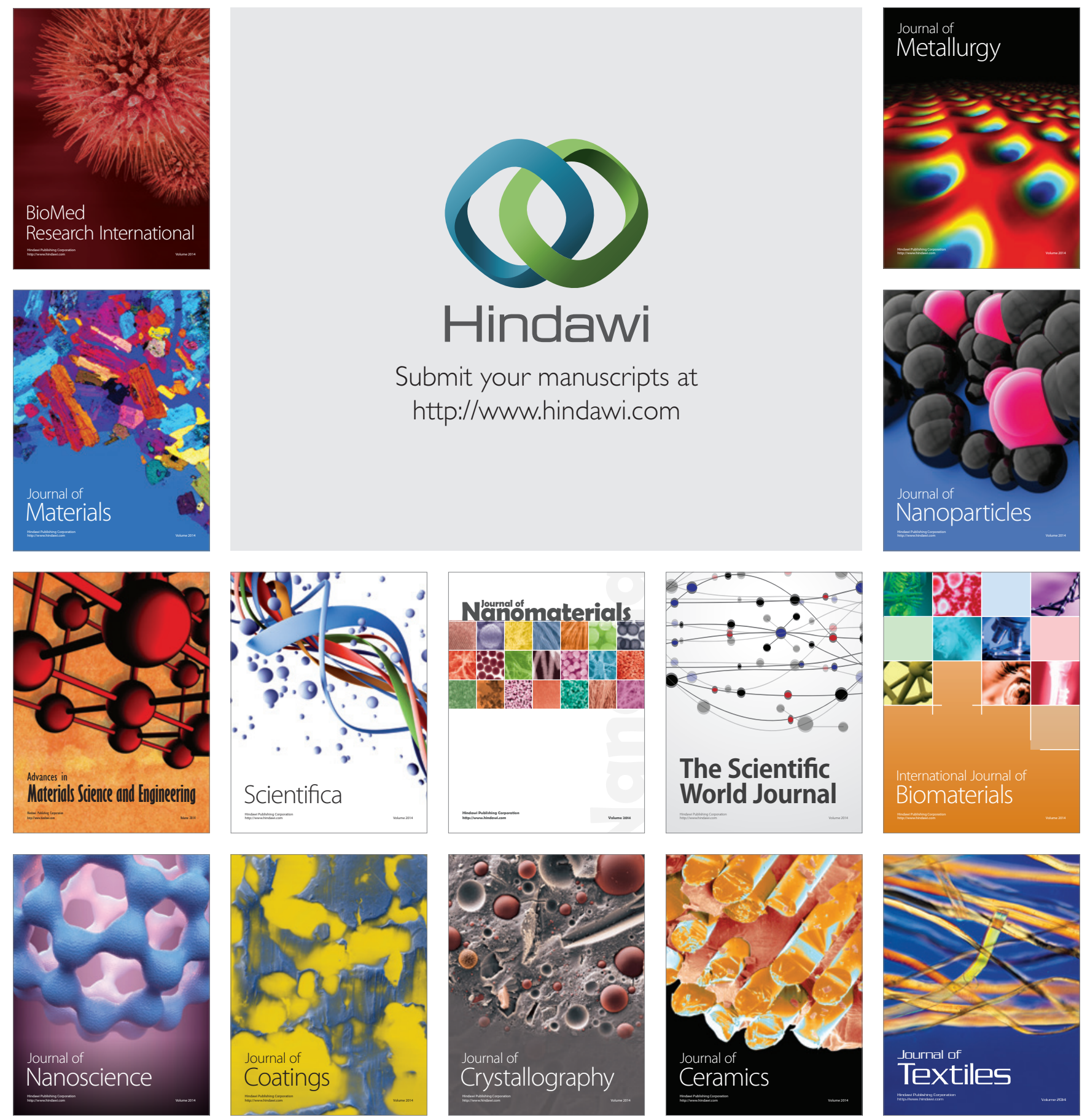